\title{
A new supersymmetric index
}

\section{Citation}

Cecotti, Sergio, Paul Fendley, Ken Intriligator, and Cumrun Vafa. 1992. "A New Supersymmetric Index." Nuclear Physics B 386 (2): 405-52. https://doi.org/10.1016/0550-3213(92)90572-s.

\section{Permanent link}

http://nrs.harvard.edu/urn-3:HUL.InstRepos:41385051

\section{Terms of Use}

This article was downloaded from Harvard University's DASH repository, and is made available under the terms and conditions applicable to Other Posted Material, as set forth at http:// nrs.harvard.edu/urn-3:HUL.InstRepos:dash.current.terms-of-use\#LAA

\section{Share Your Story}

The Harvard community has made this article openly available.

Please share how this access benefits you. Submit a story.

Accessibility 
HUTP-92/A021

SISSA 68/92/EP

BUHEP-92-14

\title{
A New Supersymmetric Index
}

\author{
Sergio Cecotti ${ }^{\dagger}$, Paul Fendley ${ }^{\star}$, Ken Intriligator ${ }^{\infty}$ and Cumrun Vafa ${ }^{\infty}$ \\ $\dagger$ International School for Advanced Studies, SISSA-ISAS \\ Trieste and I.N.F.N., sez. di Trieste, Trieste, Italy \\ *Department of Physics, Boston University \\ 590 Commonwealth Avenue, Boston, MA 02215, USA \\ ${ }^{\infty}$ Lyman Laboratory of Physics \\ Harvard University, Cambridge, MA 02138, USA
}

\begin{abstract}
We show that $\operatorname{Tr}(-1)^{F} F e^{-\beta H}$ is an index for $N=2$ supersymmetric theories in two dimensions, in the sense that it is independent of almost all deformations of the theory. This index is related to the geometry of the vacua (Berry's curvature) and satisfies an exact differential equation as a function of $\beta$. For integrable theories we can also compute the index thermodynamically, using the exact $S$-matrix. The equivalence of these two results implies a highly non-trivial equivalence of a set of coupled integral equations with these differential equations, among them Painleve III and the affine Toda equations.
\end{abstract}




\section{Introduction}

There has been much progress in understanding supersymmetric quantum field theories in the last decade. Supersymmetry turns out to be a strong symmetry principle which allows one to get a firm grip on certain aspects of these theories. For example, Witten's index $\operatorname{Tr}(-1)^{F} e^{-\beta H}$ [1] is an effective tool in addressing questions of supersymmetry breaking. It is natural to ask if there are other 'index-like' objects which can be computed exactly and provide further insight into the structure of supersymmetric theories. The aim of this paper is to show in two-dimensional $N=2$ supersymmetric theories, there is such an object: $\operatorname{Tr}(-1)^{F} F e^{-\beta H}$. We call this an index because it is independent of almost all deformations of the action. It, however, does depend on a finite set of (relevant or marginal) perturbations in a way which can be computed exactly.

Supersymmetric theories in two dimensions are among the simplest quantum field theories. Two-dimensional conformal theories with $N=2$ supersymmetry can be used to construct string vacua and have thus been studied extensively recently. All $N=2$ theories in two dimensions, whether or not they are conformal, have a set of observables, the (supersymmetric) chiral fields, which form a ring under operator product. This is called the chiral ring [2] (for a review see [3]). This ring can be computed exactly using the techniques of topological field theories [4] (see also [5,6] ) as all the $N=2$ theories have a topological counterpart (called the 'twisted version'). The study of chiral rings turn out to be a very powerful tool in unravelling the geometry of the vacua of the supersymmetric theory. In particular by joining the topological and anti-topological versions of $N=2$ theories, one can derive (integrable) differential equations ( $t t^{*}$ equations) to compute the Berry's curvature for the vacuum bundle of the supersymmetric theory as one perturbs the $N=2$ theory [3] (see also [7:8] ). It was observed in [3] that the solutions of these equations resemble a kind of partition function for kinks of the theory. This, however, remained a somewhat mysterious connection to be explained. In this paper we will see that these computations are related to the new index $\operatorname{Tr}(-1)^{F} F e^{-\beta H}$ which encodes aspects of the spectrum and the interactions of the kinks. In particular, the $t t^{*}$ equations provide an exact differential equation in $\beta$ for the new index for any $N=2$ theory.

These somewhat formal derivations can be checked very explicitly in many special cases. In particular when the $N=2$ theory is integrable, the existence of infinitely-many conserved charges allows one to construct the $S$-matrix (more or less uniquely) [9]. In such cases, one can use the exact $S$-matrix to find integral equations for the non-perturbative 
partition function $\operatorname{Tr} e^{-\beta H}$. This powerful method is known as the thermodynamic Bethe ansatz (TBA) 10. In particular, the TBA analysis for a large class of $N=2$ integrable theories in two dimensions was carried out in [11,12], confirming the conjectured $S$-matrices as in particular reproducing the correct central charges in the UV limit. One can extend the usual TBA analysis by allowing arbitrary chemical potentials, and in particular one can compute objects such as $\operatorname{Tr} e^{i \alpha F} e^{-\beta H}$. This allows us, as a special case, to compute $\operatorname{Tr}(-1)^{F} F e^{-\beta H}$ in these theories in terms of integral equations.

Thus for integrable theories we seem to have two inequivalent methods to compute the new index: one in terms of differential equations characterizing the geometry of the vacuum bundle, and the other in terms of coupled integral equations coming from TBA. It is a highly non-trivial check on all these ideas that the solutions to these equations are the same. We have checked this using numerical solutions to both systems of equations. Due to the non-linearity of our differential equation and complexity of the coupled integral equations, we have not been able to show directly (i.e., analytically) that these are the same. In fact, turning things around, physics has predicted a surprising equivalence between coupled integral equations and certain differential equations (such as radial affine toda equations), a result which is yet to be proven mathematically!

The organization of this paper is as follows:

In section 2 we introduce the new index, and discuss in what sense it is an index (i.e., we see that it is independent of $D$-term perturbations). The derivation of this result in this section is very simple but unfortunately requires a certain formal manipulation which is not always easy to rigorously justify. In section 3 we discuss the geometry of vacua and review the results of [3]. Here we show how to rephrase our new index as a computation in the geometry of vacua. In particular we show why our index depends only on $F$-terms, thus giving a more rigorous derivation of the results of section 2. Moreover, this allows us to effectively compute the new index in terms of solutions of certain non-linear differential equations.

In section 4 we discuss the infra-red expansion of the index. We show in particular that at least the leading term (the one-particle contribution) and the next leading term (the two-particle contribution) are universal. This means that they just depend on the mass and the central term of the supersymmetry algebra and the allowed soliton configurations. In section 5 we review briefly the results of [11] and discuss how the new index can be computed for integrable theories using the TBA. In section 6 we consider a number of examples including $N=2$ sine-Gordon and minimal $N=2$ theories perturbed by least and 
most relevant perturbations. We write down the differential equations and the integral equations which are presumably equivalent. We explicitly check this for some of the examples numerically. Moreover in this section we use the TBA to compute the more general object $\operatorname{Tr}(-1)^{F} F^{l} e^{-\beta H}$ and show that, for $l>1$ it is not an index and it does depend on the choice of D-terms, as expected.

In section 7 we present our conclusions. Finally in appendix $\mathrm{A}$ the $t t^{*}$ equations are rederived, in a quick but somewhat non-rigorous way in the same spirit as the arguments in section 2 .

\section{2. $\operatorname{Tr}(-1)^{F} F e^{-\beta \mathrm{H}}$}

In this section we discuss the existence of a new supersymmetric 'index' for $N=2$ supersymmetric quantum field theories in two dimensions. Our emphasis in this section is just on formulating what this index is; in the following sections we show how it may be computed.

Let us start with Witten's index $\operatorname{Tr}(-1)^{\mathrm{F}} \mathrm{e}^{-\beta \mathrm{H}}$ [1]. This index is defined for $N \geq 1$ supersymmetric theories in any dimension. It is an index because it is independent of finite perturbations of the theory, provided the space is compact and does not break supersymmetry (e.g., a $d$-dimensional torus). The idea is simply that there are two types of states in the Hilbert space: states which come in pairs $|s\rangle, Q|s\rangle$ where $Q$ is the supersymmetry charge with $Q^{2}=H$, and states which come isolated, i.e., the ones which are annihilated by $Q$ and are ground states of the theory with $H=0$. The pairs, which necessarily have $H \neq 0$, do not contribute to the Witten index as they have opposite $(-1)^{F}$. This follows from the fact that $\left\{(-1)^{F}, Q\right\}=0$. Therefore this index simply counts the ground states of the theory weighted with \pm 1 , depending on the parity of $(-1)^{F}$. Any finite perturbation of the theory does not change this index: if massive states become ground states they must do so in pairs, so one adds $\mathrm{a}+1$ and $\mathrm{a}-1$ to the index. Similarly, the only way a ground state can become massive is for ground states with opposite $(-1)^{F}$ to pair up, so again the net contribution to the index is zero. This index has been a powerful object in probing questions of supersymmetry breaking in supersymmetric theories.

It is well known that the above argument does not apply for non-compact spaces. Consider a Hilbert space based on $R^{d}$. The above argument breaks down in this case because the eigenvalues of $H$ are typically continuous. In particular it may not be true 
that the density of states for $|s\rangle$ and $Q|s\rangle$ are equal. The contribution to the index may be written as

$$
(-1)^{f} \int d E\left(g_{+}(E)-g_{-}(E)\right) e^{-\beta E}
$$

where $g_{ \pm}(E)$ are the density of states distributions for $|s\rangle$ and $Q|s\rangle$ and these states contribute $\pm(-1)^{f}$ to the index. If $g_{+}-g_{-}$is nonzero for $E \neq 0$, the contribution of massive states to the index does not vanish and thus as we change the parameters in the theory the index changes. In particular it does depend on $\beta$. Examples of this phenomena have been found in some simple quantum-mechanical systems with $N=1$ supersymmetry [13] where it can be computed exactly using the Callias-Bott-Seeley index theorem [14].

Let us consider this situation for the $N=2$ supersymmetric theories in $d=2$ where we take space to be the real line. The $N=2$ supersymmetry algebra on the real line can be written as

$$
\begin{gathered}
Q^{+2}=Q^{-2}=\bar{Q}^{+2}=\bar{Q}^{-2}=\left\{Q^{+}, \bar{Q}^{-}\right\}=\left\{Q^{-}, \bar{Q}^{+}\right\}=0 \\
{\left[F, Q^{ \pm}\right]= \pm Q^{ \pm} \quad\left[F, \bar{Q}^{ \pm}\right]=\mp \bar{Q}^{ \pm}} \\
{\left[Q^{ \pm}, H_{L, R}\right]=\left[\bar{Q}^{ \pm}, H_{L, R}\right]=\left[F, H_{L, R}\right]=0} \\
\left\{Q^{+}, Q^{-}\right\}=H_{L} \quad\left\{\bar{Q}^{+}, \bar{Q}^{-}\right\}=H_{R} \\
\left\{Q^{+}, \bar{Q}^{+}\right\}=\Delta \quad\left\{Q^{-}, \bar{Q}^{-}\right\}=\bar{\Delta}
\end{gathered}
$$

where $H_{L, R}=H \pm P$, and $\Delta$ is a $c$-number which is the central term of the supersymmetry algebra. The fermion number $F$ is the charge corresponding to the global $O(2)$ symmetry of $N=2$ supersymmetric theories. We also have $\left(Q^{+}\right)^{\dagger}=Q^{-}$and $\left(\bar{Q}^{+}\right)^{\dagger}=\bar{Q}^{-}$. Defining $Q_{ \pm}=(1 / \sqrt{2})\left(Q^{ \pm}+\bar{Q}^{ \pm}\right)$we have

$$
\left\{Q_{-}, Q_{+}\right\}=H \quad\left[Q_{ \pm}, H\right]=0
$$

It is well known that on a non-compact space in general the central term $\Delta$ can be non-zero [15]. $\Delta$ depends on the boundary conditions at spatial infinity: with multiple vacua, we have the freedom of having different boundary conditions at left-right spatial infinity and thus different central terms. Let us denote the vacuum at left spatial infinity by $a$ and the one on the right by $b$, so that the central term in the above algebra may be labeled by $\Delta_{a b}$. Having multiple vacua allows kinks which interpolate from one vacuum at left spatial infinity to another one at right spatial infinity. The kinks we will denote by 
$k_{a b}$. In general, such kinks (even the lowest-energy configuration in each sector) may be stable or unstable. We can, however, derive a lower bound on the mass of any kink. In the $a b$ sector the positivity of $\left\{A, A^{\dagger}\right\}$ where $A=\left(H_{R} \bar{\Delta}\right)^{1 / 2} Q^{+}-\left(H_{L} \Delta\right)^{1 / 2} \bar{Q}^{-}$implies the Bogomolnyi bound $E^{2}-P^{2} \geq\left|\Delta_{a b}\right|^{2}$. A kink $k_{a b}$ or, more generally, any state in the $a b$ sector must therefore have mass $m \geq\left|\Delta_{a b}\right|$.

We now ask whether Witten's index is a good index for the $N=2$ case on open space. Consider varying the Hamiltonian of the theory, respecting $N=2$ supersymmetry. We wish to compute

$$
\delta \operatorname{Tr}(-1)^{F} e^{-\beta \mathrm{H}}=-\beta \operatorname{Tr}(-1)^{F} \delta \mathrm{H} e^{-\beta H}
$$

Using (2.3) we can write this ast

$$
\begin{gathered}
-\beta \operatorname{Tr}(-1)^{F} \delta\left\{Q_{+}, Q_{-}\right\} e^{-\beta \mathrm{H}} \\
=-\beta \operatorname{Tr}(-1)^{F}\left(\left\{\delta Q_{+}, Q_{-}\right\}+\left\{Q_{+}, \delta Q_{-}\right\}\right) e^{-\beta H}
\end{gathered}
$$

Each of the above terms vanishes. To see this note that whenever we are computing

$$
\operatorname{Tr}(-1)^{F}\{A, B\} O
$$

where $A$ and $B$ are fermionic and where at least one of them commutes with $O$, we formally get zero. Suppose $A$ commutes with $O$. Then for the $A B$ term contributing to the above trace, we can take $A$ around the trace, because the trace is cyclic. The term picks up a minus sign because $A$ anticommutes with $(-1)^{F}$. This leaves $-B A$, which cancels $+B A$ from the other term in the anti-commutator. The same argument works if $A$ and $B$ are bosonic operators, and we replace anti-commutators with commutators. We shall refer to this as the $A B$ argument. For this formal argument to be actually valid one needs to put restrictions on the nature of the operators $A$ and $B$, which we assume to be satisfied in our case日 [16]. That the $A B$ argument is valid in our case is confirmed in the next section where we derive the results of this section without making use of this assumption.

Applying these general statements to the above variation of the index, where in one term $Q_{-}$and in the other term $Q_{+}$plays the role of $A$ in the $A B$ argument, we find that

1 We have been somewhat cavalier with regard to boundary conditions at spatial infinity in taking the variations. This point is elaborated upon in the appendix.

2 In the supersymmetric quantum-mechanical version of this statement, this can be explicitly checked to be true, where $A$ (after being dressed by $O$ ) is a trace-class operator and $B$ (after being dressed by $O$ ) is bounded. 
the variation of Witten's index is zero for $N=2$ theories, and it is thus a good index even for non-compact space. This in particular means that in sectors where the left and right vacua are not the same $\operatorname{Tr}(-1)^{F} e^{-\beta H}$ vanishes: we are free to take $\beta$ large because it is an index, and since the ground state in this sector has non-zero energy (because it interpolates between two distinct vacua) we get zero.

For an $N=1$ supersymmetric theory in two dimensions the fermion number $F$ is only defined $\bmod 2$. However, in a two-dimensional $N=2$ theory there is a $U(1)$ fermionnumber charge, because the fermions are complex. Given the power of Witten's index in understanding the structure of supersymmetric quantum field theories, it is thus natural to ask what kinds of objects may be of interest when we have this additional charge. The most natural thing to consider would be

$$
Z(\alpha, \beta)=\operatorname{Tr} e^{i \alpha F} e^{-\beta H}
$$

At $\alpha=\pi$ this is just Witten's index. For $\alpha=0$ it is just the standard partition function of the theory, so we expect $Z(0, \beta)$ to be the extreme opposite to an 'index', as it should depend on every little detail of the theory. So let us go back to the point $\alpha=\pi$ and just move slightly away. In other words, consider

$$
I_{l}(\beta)=\left.\frac{\partial^{l} Z(\alpha, \beta)}{\partial(i \alpha)^{l}}\right|_{\alpha=\pi}=\operatorname{Tr}(-1)^{F} F^{l} e^{-\beta H} .
$$

Needless to say, we should not expect all $I_{l}$ to be indices as that would enable us to reconstruct $Z$ itself. But maybe some of them are! In particular, consider $I_{1}=$ $\operatorname{Tr}(-1)^{F} F e^{-\beta H}$. Among all $I_{l}$ with $l \geq 1$, we will show that this and only this is a new 'index'.

To define what we mean by 'index', we must recall that there are two distinct ways to perturb an $N=2$ supersymmetric theory in two dimensions [17]: $D$-terms and $F$-terms. In general, the $D$-terms can be written as integrations of superfields over the full superspace $d^{4} \theta$ and the $F$-terms which are integration of chiral and anti-chiral fields over half the superspace $d^{2} \theta^{+}$and $d^{2} \theta^{-}$respectively. Chiral fields commute with $Q^{+}$and $\bar{Q}^{+}$and anti-chiral fields commute with $Q^{-}$and $\bar{Q}^{-}$. The $\operatorname{Tr}(-1)^{F} F e^{-\beta \mathrm{H}}$ is independent of the $D$-terms and in this sense it is an index. It however, does depend on the $F$-terms. In order to explain why we use the word 'index' when it does depend on $F$-terms it is convenient to consider the following interesting class of examples of $N=2$ supersymmetric QFT's. Consider 2d supersymmetric sigma models with target space being 
a Kahler manifold $M$. This gives an $N=2$ supersymmetric theory [18]. Any variation of the metric of $M$ respecting the complex structure of $M$ and the Kahler class of the metric (i.e., leaving the integral of the Kahler form on the two-cycles unchanged) can be written as a $D$-term and so does not affect our index. In fact this includes essentially all possible perturbations of the manifold, modulo variations of complex structure and Kahler structure which usually form a finite dimensional space of perturbations. So it is with this kind of example in mind that we call the above object an 'index'. Another interesting class of $N=2$ supersymmetric theories is provided by Landau-Ginzburg theories. In these cases the superpotential $W$ is the $F$-term and it has only a finite number of perturbations which do not change the behavior of potential at infinity in field space. These turn out to be the relevant (and marginal) perturbations. The index depends only on $W$.

Here we show that $\operatorname{Tr} F(-1)^{F} e^{-\beta H}$ does not depend on the $D$-terms. The variation of the $D$-term can be written as inserting $\left\{Q^{+},\left[\bar{Q}^{-}, \Lambda(x)\right]\right\}$ in the path integral where $\Lambda$ itself can be written as $\left\{Q^{-},\left[\bar{Q}^{+}, K\right]\right\}$. This follows from the fact that the $D$-term comes from integration over all four Grassman coordinates. The path-integral is over an infinite cylinder of perimeter $\beta$ with the above term inserted at all points $x$ and integrated over the cylinder. Let us denote by $\Lambda$ the integral of $\Lambda(x)$ over space. Since $F$ commutes with both $\Lambda$ and the Hamiltonian we find that the integration of $\Lambda$ over the perimeter simply introduces an irrelevant factor of $\beta$ which can be ignored. So we can write the variation of our index as (proportional to)

$$
\delta=\operatorname{Tr}(-1)^{F} F\left\{Q^{+},\left[\bar{Q}^{-}, \Lambda\right]\right\} e^{-\beta \mathrm{H}}
$$

We are almost ready to apply the $A B$ argument, using $Q^{+}$as our $A$. This works fine, except for the fact that as we try to take $Q^{+}$around the trace, since it does not commute with the $F$, we pick up a commutator term

$$
\delta=\operatorname{Tr}(-1)^{F}\left[F, Q^{+}\right]\left[\bar{Q}^{-}, \Lambda\right] e^{-\beta \mathrm{H}}=\operatorname{Tr}(-1)^{F} Q^{+}\left[\bar{Q}^{-}, \Lambda\right] e^{-\beta \mathrm{H}}
$$

Now we can apply an argument similar to the $A B$ argument, by taking $\bar{Q}^{-}$in the term $\bar{Q}^{-} \Lambda$ around the trace. Here we pick up two minus signs, and so we get back $\Lambda \bar{Q}^{-}$which thus cancels the second term in the commutator and we get zero, as was to be shown.

The $A B$ argument does not allow us to show that any of the other $I_{l}$ are independent of $D$-term perturbations 3 . In fact, in a free massive $N=2$ theory, it is easy to compute $I_{l}$;

3 One can show using the $A B$ argument that the $D$-terms will not affect the one-particle contribution of Bogomolnyi-saturated states to $I_{l}$, in accordance with the fact that the mass and fermion number of these states are independent of $D$-terms. 
the $I_{2 l}$ are non-vanishing and for $l \neq 0$ they do depend on the mass of the particle, which in turn depends on the $D$-term. In section 6 we consider other examples for which all $I_{l}$ are non-vanishing and all of them for $l>1$ depend on the $D$-term. Therefore $I_{1}$ is the only additional index that exists other than Witten's index. From now on we will refer to the new index simply as $I$, dropping the subscript 1 .

Our index is actually a matrix, because we have to fix the boundary condition at spatial infinities to be vacua of the theory. If we choose the left vacuum to be $a$ and the right one to be $b$, we have the index $I$ as a matrix

$$
I_{a b}=\operatorname{Tr}_{a b}(-1)^{F} F e^{-\beta H}
$$

One has to be careful about what we mean by $(-1)^{F}$. In general all that is required from this operator is that it anti-commute with fermionic fields. In our case, as we have mentioned before, since $F$ is in fact well defined as an operator, one can just define $(-1)^{F}=e^{i \pi F}$. Note, however this operator no longer squares to one. The reason for this is that in the $(a b)$ sector the vacuum will in general have a non-integral fermion number $f_{a b}$. This phenomenon is well known 19,20,21]. Only the fermion number relative to that of the vacuum is integral. Using this fact and the hermiticity of $H$ and $F$ we can write

$$
I_{a b}= \pm e^{i \pi f_{a b}}\left|I_{a b}\right|
$$

$C P T$ invariance puts constraints on our index. $C P T$ takes a state in the $(a b)$ sector to one in $(b a)$ sector, and it takes fermion number $F$ to $-F$. In particular, $C P T$ invariance requires $f_{a b}=-f_{b a}$, and therefore

$$
I_{a b}=-I_{b a}^{*}
$$

There is no fractional fermion number in $(a a)$ sector: the fermion number is additive, i.e., $f_{a c}=f_{a b}+f_{b c}$, implying that $f_{a a}=f_{a b}+f_{b a}=0$. We see from (2.5) that $I_{a a}$ is real, and from (2.6) it follows that

$$
I_{a a}=0
$$

Note that if we had defined $(-1)^{F}=e^{(2 n+1) i \pi F}$ then the index would have changed by a phase $I_{a b} \rightarrow e^{2 i n \pi f_{a b}} I_{a b}$. However, under this ambiguity, the eigenvalues of $I$ are unambiguous. Because of the additivity of the fractional part of the fermion number, and since $f_{a b}=-f_{b a}$, we can write $f_{a b}=f_{a}-f_{b}$; a change of basis $b \rightarrow e^{2 i n \pi f_{b}} b$ gets rid of the phase without changing the eigenvalues. 
In fact, we can do better; we can get rid of all the phases (modulo \pm ) of our matrix by changing the basis $b \rightarrow e^{i \pi f_{b}} b$. In this way we find that $I$ is a purely real matrix, and the condition (2.6) implies that it is anti-symmetric. Thus its eigenvalues are either zero, or (purely imaginary) complex-conjugate pairs. To make the eigenvalues real, we define the $Q$-index to be

$$
Q_{a b}=\frac{i \beta}{L} \operatorname{Tr}_{a b}(-1)^{F} F e^{-\beta H},
$$

where $L$ is the volume of the space. With this definition, $Q$ is a hermitian matrix with real eigenvalues, such that non-zero eigenvalues come in pairs of opposite sign. To see the reason we divided by $L$ in the definition of $Q$, consider $Z(\alpha, \beta)$ (from (2.4)) with boundary conditions at infinity corresponding to a normalized eigenstate of $Q_{a b}$. Because it is an extensive thermodynamic quantity, $\ln Z(\alpha, \beta)=\ln \operatorname{Tr}\left(e^{i \alpha F} e^{-\beta H}\right)$ is proportional to $L$ as $L \rightarrow \infty$. Therefore

$$
\left.\frac{\beta}{L} \partial_{\alpha} \log \operatorname{Tr}\left(e^{i \alpha F} e^{-\beta H}\right)\right|_{\alpha=\pi}=\frac{\frac{i \beta}{L} \operatorname{Tr} F(-1)^{F} e^{-\beta H}}{\operatorname{Tr}(-1)^{F} e^{-\beta H}}=Q,
$$

where the denominator is not proportional to $L$, because it is Witten's index, and can be chosen to be 1 in an orthonormal basis of eigenstates of $Q$. Thus we see that $Q$ as defined above is well-defined as $L \rightarrow \infty$.

Usually the contribution of $n$ kinks (particles) to a partition function is proportional to $L^{n}$. One may incorrectly conclude from this that only the one-kink states contribute to $Q$. In fact we will see in later sections that the $n$-kink contributions to $I$ do not generally vanish and are proportional to $L$. The contribution comes from regions where all the kinks are near each other and the factor of $L$ is associated with the center of mass. It can be seen that any configuration where one of the kinks is very far from the rest does not contribute to $Q$ : the contribution factorizes and at least one piece will simply be the contribution to Witten's index from massive kinks, which vanishes. One can also see this from the path-integral computation where the exact fermion zero modes associated to each kink when they are far away cannot be absorbed by one $F$.

In the next section we will see that $Q$ is the same as the matrix element of the chiral fermion number:

$$
Q_{a b}=\left\langle a\left|Q^{5}\right| b\right\rangle
$$

Using this expression along with the hermiticity of $Q^{5}$, and noting that $C P T$ changes the sign of $Q^{5}$, we again see that the eigenvalues of $Q$ are real and symmetrically located relative to zero. 
For the remainder of this section, we will discuss the kind of states in the Hilbert space which contribute to our index. In general, there are three types of irreducible representations of the supersymmetry algebra (2.2). The generic irreducible representation of (2.2) is four-dimensional, with a definite eigenvalue for $E$ and $P$ (as $H_{L, R}$ commute with everything). This follows from the fact that the four supersymmetry charges which generate the algebra are pairwise adjoint of one another and have $c$-number anticommutators. We can generate the representation by taking $Q^{+}$and $\bar{Q}^{-}$as 'creation' operators acting on a state which is annihilated by the 'annihilation' operators $Q^{-}$and $\bar{Q}^{+}$:

$$
|s\rangle \quad Q^{+}|s\rangle \quad \bar{Q}^{-}|s\rangle \quad Q^{+} \bar{Q}^{-}|s\rangle
$$

When $E^{2}-P^{2}=\Delta \bar{\Delta}$, i.e., if the state saturates the Bogomolnyi bound, then it is well known [15] that this representation is reducible: $\left.A=\left(H_{R} \bar{\Delta}\right)^{1 / 2} Q^{+}-\left(H_{L} \Delta\right)^{1 / 2}\right) \bar{Q}^{-}$and its adjoint anticommute, and so both must annihilate $|s\rangle$. This leaves us with the reduced supersymmetry multiplet

$$
|s\rangle \quad Q^{+}|s\rangle
$$

Finally, for $E=P=\Delta=0$ this representation is further reduced to the trivial representation. This representation only appears for the $(a a)$ sectors, and are the only states which contribute to Witten's index in this sector. However, because $I_{a a}=0$ these states are not relevant for the new index $I$.

At first glance, one might think that only the reduced multiplets contribute to our index. If $|s\rangle$ has fermion number $f$, a non-reduced multiplet (2.10) naively contributes (up to an overall phase) $(f-2(f+1)+(f+2)) e^{-\beta E}=0$, whereas a reduced multiplet (2.11) contributes (up to an overall phase) $(f-(f+1)) e^{-\beta E}=-e^{-\beta E}$. Thus it appears that $I$ receives contributions only from Bogomolnyi-saturated states, which are simply the one-soliton subsectors. This argument is incorrect, for the same reason that the naive argument which states that $\operatorname{Tr}(-1)^{F} e^{-\beta H}$ is independent of $\beta$ is not in general valid when $H$ has a continuous spectrum, as is the case in non-compact spaces. Formally, we have deduced the vanishing of the contribution of the non-reduced multiplets only when the spectrum of the Hamiltonian is discrete. When it is continuous, as with a model on a real line, we have to deal with the density of states of the non-reduced multiplets; they are not necessarily equal and do not necessarily cancel in computing $I$. We may wish to regularize the theory by putting it in a box of size $L$ and then take $L \rightarrow \infty$. In order to recover the soliton sector $a b$, the field configurations on the left and the right of the box (in this 
case just a line interval) cannot be the same. Thus we cannot impose periodic boundary conditions. We must compute the object in finite but not periodic box, and this breaks the supersymmetry. The spectrum is discrete in this case, but without supersymmetry the naive argument no longer holds. Thus for a finite box we may get contribution from non-reduced supersymmetry multiplets to the index $Q$ in the sector $a b$ with $a \neq b$. This may persist even when the size $L \rightarrow \infty$ 日. Thus we are computing a kind of 'anomaly', which remains after the regulator is removed.

Let $g_{f}(E)$ be the density of states for $|s\rangle, 2 g_{f+1}(E)$ be the density of states for states spanned by $Q^{+}|s\rangle$ and $\bar{Q}^{-}|s\rangle$, and $g_{f+2}(E)$ be the density of states for states $Q^{+} \bar{Q}^{-}|s\rangle$. We should thus not expect the continuum densities $g_{f}(E), g_{f+1}(E)$, and $g_{f+2}(E)$ to be equal in the $(a, b)$ sector of the theory with $a \neq b$. Recall, though, that we proved using $N=2$ supersymmetry that the contribution to Witten's index from these states must cancel. This means that we must have

$$
g_{f}(E)+g_{f+2}(E)=2 g_{f+1}(E)
$$

since the states on the two sides make opposite contributions to Witten's index. Using (2.12) we see that the contribution of the four dimensional representation to the index $I$ in the $(a, b)$ sector is of the form

$$
e^{i \pi f} \int d E\left(g_{f+2}(E)-g_{f}(E)\right) e^{-\beta E}
$$

We will see explicitly how this is generically nonzero in the following sections.

\section{Geometry of Ground States and the New Supersymmetric Index}

In this section we review some aspects of the work done in [3] which are useful for the considerations of this paper. In particular, we show why the 'Q'-matrix discussed there is in fact the new supersymmetric index given by $\operatorname{Tr}(-1)^{F} F e^{-\beta H}$ discussed in the previous section. It is convenient to exchange the role of space and time (i.e., do a 'modular transformation') and take the space to be a circle (with perimeter $\beta$ to correspond to the index computation), with periodic boundary conditions. Time is now a line of length $L$.

\footnotetext{
4 This is indeed one way that the $\beta$-dependence of $\operatorname{Tr}(-1)^{F} e^{-\beta H}$ has been computed in the supersymmetric quantum mechanics examples [22], which is related to the Callias-Bott-Seeley index.
} 
Consider an arbitrary $N=2$ supersymmetric quantum field theory in two dimensions. From (2.3) and the positivity of the inner product together with the fact that $Q_{-}=Q_{+}^{\dagger}$, it is easy to show that the ground states of the theory are characterized by

$$
H|a\rangle=0 \leftrightarrow Q_{ \pm}|a\rangle=0
$$

There is thus a one-to-one correspondence between the ground states of the theory and the $Q_{+}$or $Q_{-}$cohomology. This cohomology is definable because each of these operators squares to zero (note that on a compact space (circle) the supersymmetry algebra has no central term and we get $\left.Q_{+}^{2}=Q_{-}^{2}=0\right)$. The analogy to keep in mind is that $Q_{+}$is like a $d$ operator acting on the differential forms on a manifold, $Q_{-}$is like the adjoint operator $d^{\dagger}$ and the ground states $|a\rangle$ are like the harmonic representative of $d$ or $d^{\dagger}$ cohomology.

In correspondence with the ground states in the Hilbert space, there are chiral operators $\phi_{i}$ in the theory defined by the condition that

$$
\left[Q_{+}, \phi_{i}\right]=0
$$

and similarly there are anti-chiral operators $\bar{\phi}_{i}$ which commute with $Q_{-}$. Acting on a vacuum by a chiral operator, we get another state which is $Q_{+}$closed, another $Q_{+}$ cohomology element. In this way the chiral fields, modulo the fields that are trivially chiral, i.e. modulo fields which are themselves $Q_{+}$(anti-)commutator, are in one-to-one correspondence with the $Q_{+}$cohomology elements and thus the ground states. If we pick a canonical ground state (to be defined below) denoted by $|0\rangle$, this can be stated as

$$
\phi_{i}|0\rangle=|i\rangle+Q_{+}|\Lambda\rangle
$$

where $|i\rangle$ denotes another ground state. Similarly we can label the ground states using the anti-chiral fields $\bar{\phi}_{i}$ which leads to the states $|\bar{i}\rangle$. The chiral fields form a ring among themselves, called the chiral ring, which is defined by

$$
\begin{gathered}
\phi_{i} \phi_{j}=C_{i j}^{k} \phi_{k}+\left[Q^{+}, \Lambda\right] \\
\phi_{i}|j\rangle=C_{i j}^{k}|k\rangle .
\end{gathered}
$$

The matrix $\left(C_{i}\right)_{j}^{k}=C_{i j}^{k}$ denotes the action of the chiral field $\phi_{i}$ on the ground states (once we ignore the components orthogonal to ground states). Similar statements apply to anti-chiral fields with $C_{i j}^{k}$ replaced by the complex conjugate quantity $\left(C_{i j}^{k}\right)^{*}$. 
We can define a symmetric metric $\eta$ and a hermitian metric $g$ among the ground states by

$$
\eta_{i j}=\langle i \mid j\rangle \quad g_{i \bar{j}}=\langle\bar{j} \mid i\rangle
$$

Note that the metric $g$ is the usual metric in the Hilbert space of the $N=2$ theory and $\eta$, which is not hermitian, is a kind of 'topological' metric. As discussed in the previous section there are two ways to perturb the action: the 'D-terms' (denoted by $K(X, \bar{X})$ below) and the 'F-terms' which are the chiral fields (now viewed as superfields) and integrated over half of the superspace:

$$
S \rightarrow S+\int d^{2} z d^{4} \theta K(X, \bar{X})+\int d^{2} z d^{2} \theta^{+} t_{i} \phi_{i}+\int d^{2} z d^{2} \theta^{-} \bar{t}_{i} \bar{\phi}_{i} .
$$

Then it is possible to show (see [3]) that the chiral ring and the metrics $\eta$ and $g$ depend only on the F-terms, i.e. they depend only on $t_{i}, \bar{t}_{i}$ and are independent of $K$. The flavor of the argument is very similar to the argument in the previous section in showing that our index is independent of $D$-terms, but it has the advantage of being rigorous.

The ring matrices $C_{i}$ and the metric $\eta$ can also be related to computations of correlation functions in a topological theory [4] corresponding to 'twisting' the $N=2$ quantum field theory and can thus be easily computed exactly [4, 5, 23, 6]. Basically the topological theory is the same as the ordinary $N=2$ theory on flat manifolds but differs from it when the two-dimensional manifold is not flat, in such a way that the charge $Q_{+}$ transforms as a scalar, and is thus a symmetry even if the space is not flat. The way this is accomplished is by introducing a background gauge field set equal to half the spin connection of the manifold, and coupling it to the fermion number current. Thus a field which previously had spin $s$ and fermion charge $q$ will now have spin $s-\frac{1}{2} q$. This in particular makes $Q_{+}$which had spin $1 / 2$ and fermion number +1 , a scalar. If $S$ denotes the action of ordinary $N=2$ theory, $S_{t}$ denotes the action for the topological theory, $j$ denotes the fermion number current, and $\omega_{\mu}$ denotes the $U(1)$ spin connection we have

$$
S_{t}=S+\frac{i}{2} \int j_{\mu} \omega^{\mu}
$$

An important property of the topological action is that the energy-momentum of the topological theory is itself $Q_{+}$trivial:

$$
T_{\mu \nu}^{t}=T_{\mu \nu}+\frac{1}{2} \epsilon_{\alpha(\mu} \partial_{\nu)} j^{\alpha}=\left\{Q_{+}, \Lambda\right\}
$$


implying that the correlation functions for chiral fields are independent of the metric. By translating the computation of the $N=2$ topological theory into the language of the ordinary $N=2$ theory, this provides exactly the quantities $\eta$ and the ring matrices $C$. The basic observation is that if we consider a hemisphere and do the path-integral in the topological theory we get a state (on the boundary circle) which is annihilated by the symmetry charge $Q_{+}$. Moreover because the energy momentum tensor is $Q_{+}$-trivial, any local variation of the data (such as the variation of the metric on the hemisphere) does not change the $Q_{+}$cohomology class of the state, and so the path integral of the topological theory leads to a well-defined state in the $Q_{+}$-cohomology, and thus to a ground state of the ordinary $N=2$ theory. In particular the state that we called the vacuum $|0\rangle$ corresponds to the state we get when we do the path-integral with no insertion of any fields on the hemisphere. Simple arguments show that $C$ and $\eta$ depend only on $t_{i}$ and not on $\bar{t}_{i}$. In other words, they are holomorphic.

Similarly, we can consider the anti-topological theory, which is obtained when we make $Q_{-}$a scalar. This is done simply by changing the sign of the background field, which shifts the spins by $s \rightarrow s+\frac{1}{2} q$. So the action for the anti-topological theory $S_{t *}$ is

$$
S_{t *}=S-\frac{i}{2} \int j_{\mu} \omega^{\mu}
$$

From the anti-topological theory we can easily compute $\eta_{\overline{i j}}$ and $\bar{C}$ which are simply the complex conjugate of the corresponding topological quantities $\eta$ and $C$.

The computation of the hermitian ground-state metric $g$ as a function of perturbation parameters $\left(t_{i}, \bar{t}_{i}\right)$ is more difficult. It turns out that by fusing the topological theory on one hemisphere with the anti-topological theory on the other hemisphere, we can find equations which characterize it [3]. This we shall call topological-anti-topological fusion, or $t t^{*}$ for brevity. One simply introduces a gauge connection such that the variation of ground states are orthogonal to the ground states themselves:

$$
D_{i}|a\rangle=\partial_{i}-A_{i}|a\rangle \quad \bar{D}_{i}|a\rangle=\bar{\partial}_{i}-\bar{A}_{i}|a\rangle
$$

This in particular means that the metric $g$ is covariantly constant

$$
D_{i} g=\bar{D}_{i} g=0
$$

and one finds the equations

$$
\left[D_{i}, D_{j}\right]=\left[\bar{D}_{i}, \bar{D}_{j}\right]=0
$$




$$
\left[D_{i}, \bar{D}_{j}\right]=-\beta^{2}\left[C_{i}, \bar{C}_{j}\right]
$$

(and some other equations which we will not need here). The perimeter of the space (circle) is $\beta$. We will give a quick (but not rigorous) derivation of the above equations in the spirit of the $A B$ argument of previous section in the appendix.

The first equation (3.3) shows that we can choose a holomorphic gauge with $\bar{A}_{i}=0$. This turns out to be the natural gauge in the topological theory. In more mathematical terminology we can say that the topological path-integral automatically gives holomorphic sections of the vacuum bundle. Using the covariant constancy of the metric we can write the metric $g$ as

$$
A_{i}=-g \partial_{i} g^{-1}
$$

and so the second equation in (3.3) becomes

$$
\bar{\partial}_{j}\left(g \partial_{i} g^{-1}\right)=\beta^{2}\left[C_{i}, g C_{j}^{\dagger} g^{-1}\right]
$$

In many examples these equations turn out to be among the celebrated equations of mathematical physics. For the $N=2$ sine-Gordon theory the above equation as a function of the scale turns out to correspond to radial solutions of the sinh-Gordon differential equation, which is a special case of Painleve III. These differential equations are always integrable, being related to a tau function. The integrability of these equations has been recently elaborated upon in [24]. Explicit numerical computations have been done for flows among conformal theories and also flows under generic perturbations away from conformal theories 25.

Among the perturbations of the $N=2$ theory, there is a special one corresponding to renormalization group flow. In particular if we denote the perimeter of the circle on which we base our Hilbert space as $e^{\tau}$, then changing $\tau$ should be equivalent to changing the coupling in the theory in some particular way. In the case of Landau-Ginzburg theories, this has the same effect on the F-terms as multiplying it by $e^{\tau}$. From the definition of connection it follows that

$$
\partial_{\tau}|a\rangle=A_{\tau}|a\rangle
$$

On the other hand it was shown in [3] that the variation of the ground states with respect to the perimeter is related to the action of the chiral fermion number charge $Q^{5}$ on the ground states by

$$
\partial_{\tau}|a\rangle=\frac{1}{2}\left(Q^{5}+n\right)|a\rangle
$$


where the above equality holds as long as we project both sides back to the ground states. Here $n$ is a number which measures the chiral anomaly of the theory (equal to the number of chiral fields in the LG theory). So we see that as far as the ground state action is concerned, in a holomorphic (topological) basis 5

$$
\frac{1}{2}\left(Q^{5}+n\right)|i\rangle=A_{\tau}{ }^{j}{ }_{i}|j\rangle=\left(-g \partial_{\tau} g^{-1}\right)_{i}{ }^{j}|j\rangle
$$

The equation (3.4) was derived in [3] in the context of Landau-Ginzburg theories. Since this is an important equation for us in this paper, we will now present a more general derivation of it.

It is convenient to work in the topological basis. Then a state $|i\rangle$ can be obtained as a result of topological path-integral on hemisphere, with insertion of the chiral field $\phi_{i}$. In view of the fact that the energy momentum tensor of the topological theory is $Q_{+}$trivial, it sounds contradictory to expect $\partial_{\tau}|i\rangle$ not to be zero (i.e., $Q_{+}$trivial). The way this comes about is by a subtle boundary term, as we will now see.

Let us denote the metric on the hemisphere by $h=e^{2 \phi} d z d \bar{z}$. In terms of $\phi$ the spin connection is $\omega=* d \phi$ and so the topological action (3.1) is

$$
S_{t}=S+\frac{i}{2} \int j \wedge d \phi
$$

Now we are interested in the variation of this action on the right hemisphere as we change $\phi$ by a constant. Varying the metric by an overall scale $\phi \rightarrow \phi+\epsilon$ has the effect of changing the perimeter by shifting $\tau \rightarrow \tau+\epsilon$. It is convenient to first do a partial integration on the second term above and write it as

$$
\int_{S_{R}} j \wedge d \phi=\oint_{S^{1}} j \phi-\int_{S_{R}} \phi d j
$$

where $S_{R}$ denotes the right hemisphere and $S^{1}$, the boundary circle of $S_{R}$, is where we base our Hilbert space. Shifting $\phi$ brings down from $S$ the trace of the energy momentum tensor, and from the topological addition the divergence of the axial current plus the variation of the boundary term, i.e.,

$$
\delta S=\int_{S_{R}}\left(T_{\mu}^{\mu}+\frac{1}{2} D_{\mu} j^{5 \mu}\right)+\frac{i}{2} \oint_{S^{1}} j
$$

5 More precisely, in a topological basis $|i\rangle$ obtained by inserting in the topological integral chiral operators $\phi_{i}$ with $\partial_{\tau} \phi_{i}=0$. Two such bases are related by a $\tau$ independent 'gauge transformation'. Under such changes of bases $g \partial_{\tau} g^{-1}$ transforms as a tensor. 
where we have used that in two-dimensional Euclidean field theory $j_{\mu}^{5}=i \epsilon_{\mu \nu} j^{\nu}$. The term integrated over the right hemisphere appears to be $Q_{+}$trivial because it is the trace of the energy momentum tensor of the topological theory. This statement is almost true, except for the fact that there is a well-known anomaly in the divergence of axial current which contributes $n / 2$ (in the LG theory $n$ is the number of fields). But now we see that the boundary term is also present, and is equivalent to the action of $Q^{5} / 2$ at the boundary (as follows from $\left.j^{5}=i(* j)\right)$. So the net effect on a state of the change of $\tau$ is given by

$$
\partial_{\tau}|a\rangle=\frac{1}{2}\left(Q^{5}+n\right)|a\rangle
$$

(as long as we compute the matrix element of both sides of the above equation among ground states). This is the equation (3.4] we wished to derive.

We have seen that the matrix elements of $Q^{5}$ among ground states of the supersymmetric theory are possible to compute, if we know $g$ (from (3.5)). Note that even though the fermion number is always conserved the chiral fermion number is conserved only at conformal points.

Since we are considering both massive and massless theories it may seem strange to see that the matrix elements of a non-conserved charge are somehow 'interesting' and related to RG-variations of ground states. Let us rephrase this by using a modular transformation. Consider the theory on a very long cylinder of length $L$ and circumference $\beta$. Let us put a ground state $|b\rangle$ at one end of the cylinder and another ground state $\langle a|$ at the other end. We denote the coordinates along the cylinder by $x$ and that along the circumference by $t$. The matrix elements of $Q^{5}$ can then be written as

$$
\left\langle a\left|Q^{5}\right| b\right\rangle=\left\langle a\left|i \oint_{S^{1}} j_{t}(0, t) d t\right| b\right\rangle
$$

where $S^{1}$ is a circle wrapped around the middle of the long cylinder. We have to take the limit $L \rightarrow \infty$ at the end in order to project onto the ground states in a natural way. It clearly does not matter where we insert the circle. So let us put the circle at any $x$, integrate over all $x$ and divide by $L$, i.e.,

$$
\left\langle a\left|Q^{5}\right| b\right\rangle=\frac{i}{L}\left\langle a\left|\int j_{t}(x, t) d x d t\right| b\right\rangle
$$

Now viewing $x$ as space, and $t$ as time, we see that $\int j_{t}(x, t) d x$ is the definition of the fermion number $F$ on the Hilbert space which is along the cylinder. Since fermion number 
is conserved, integrating along $t$ will just introduce an additional factor of the circumference of the cylinder $\beta$. In other words we have

$$
\int j_{t}(x, t) d x d t=\beta F
$$

So we have finally

$$
Q_{a b}=\left\langle a\left|Q^{5}\right| b\right\rangle=\frac{i \beta}{L} \operatorname{Tr}_{a b}(-1)^{F} F e^{-\beta H}
$$

where the $\operatorname{Tr}_{a b}$ means that we are taking the boundary conditions on the left and right to correspond to $\langle a|$ and $|b\rangle$ vacua. This is the new index discussed in the previous section! What is surprising is that the index can be computed exactly in terms of $g$, and $g$ is determined exactly by the differential equations (3.3). In particular we see from (3.5) that the index is given by

$$
Q_{a b}=\frac{i \beta}{L} \operatorname{Tr}_{a b}(-1)^{F} F e^{-\beta H}=-\left(\beta g \partial_{\beta} g^{-1}+n\right)_{a b}
$$

where we have used $2 g \partial_{\tau} g^{-1}=\beta g \partial_{\beta} g^{-1}$ which follows because, by scaling, we can set $\beta=e^{\tau / 2+\tau^{*} / 2}$. Often it is difficult to compare the topological basis for ground states with the path-integral choice emphasized in the previous section and more natural from the viewpoint of kinks. In such cases it is convenient to compare the eigenvalues of the $Q$ matrix on both sides of the above equation.

Note that the matrix $Q$, since it can be written solely in terms of $g$, depends only on the knowledge of the $F$-term and is independent of the $D$-terms in accord with our proof in the previous section. Our final formula, (3.8), expresses the new supersymmetric index in terms of the geometry of supersymmetric ground states. Because the curvature of this space is determined simply from the chiral ring structure constants using (3.3), the index will be an exact solution of a differential equation whose form is determined simply by the chiral ring. In other words, though our index is not purely topological, its flow in $\beta$ is determined using only topological data, namely the chiral ring.

At the conformal point, where chiral fermion number is conserved, $Q$ measures the chiral charge of Ramond vacua, i.e., the left-moving fermion number plus the right moving fermion number. In this case the state with highest charge has $Q=\hat{c}$ where $\hat{c}$ is the central charge of the $N=2$ superconformal theory [2,26]. So off criticality each eigenvalue of the $Q$ matrix, and in particular the highest one, is a kind of a generalization of a $c$-function [7] (which has no direct relationship with Zamolodchikov's definition [27], as discussed in [3]). 


\section{The infra-red expansion of $\operatorname{Tr} F(-1)^{F} e^{-\beta H}$}

In section 2 we discussed which states in the Hilbert space contribute to $\operatorname{Tr} F(-1)^{F} e^{-\beta H}$. In this section we show how to calculate the one- and two-particle contributions. These are the leading terms in infra-red limit where $\beta>>1$. We will see the simple but non-trivial nature of our index. These results must be the leading infra red behavior of the $t t^{*}$ differential equations of the previous section.

Let us start with the contribution of one-particle (kink) states to the index. In order to calculate the density of states, we put the system in a box of length $L$ with the $a$ and $b$ boundary conditions at the end of the box. To obtain a non-vanishing contribution to the index, recalling (2.7), we take $a \neq b$; in particular, we do not want periodic boundary conditions. The allowed momenta of a particle in a box are quantized as $p=n \pi / L$, where $n$ is a positive integer. Thus the density of states for each component of a supersymmetry multiplet is the same and given by $g(E) d E=L d p / \pi$. From relation (2.13), we see that one-particle states in four-dimensional multiplets do not contribute to the index; a single particle contributes if and only if it is part of a reduced supersymmetry multiplet. This in particular means that its mass should saturate the Bogomolnyi bound $m_{a b}=\left|\Delta_{a b}\right|$. So the one-particle contribution to $Q_{a b}=\frac{i \beta}{L} \operatorname{Tr}_{a b}(-1)^{F} F e^{-\beta H}$ from a kink multiplet with fermion number $\left(f_{a b}, f_{a b}+1\right)$ is given by

$$
\begin{aligned}
& i \beta\left(f_{a b}-\left(f_{a b}+1\right)\right) e^{i \pi f_{a b}} \int_{0}^{\infty} \frac{d p}{\pi} e^{-\beta \sqrt{p^{2}+m_{a b}^{2}}} \\
= & -i\left|\Delta_{a b}\right| \beta e^{i \pi f_{a b}} \frac{1}{\pi} K_{1}\left(\left|\Delta_{a b}\right| \beta\right),
\end{aligned}
$$

where $K_{1}$ is a Bessel function. This simple statement explains and makes precise the observation made in [3] that in the infra-red the Q-matrix is a kind of partition function of the solitons of the theory. The fact that the leading term in the infra-red limit is proportional to $K_{1}$ follows easily from the $t t^{*}$ equations (see appendix $B$ of [3]).

The next-leading contribution in the infrared to the index comes from the two-particle states. A two-particle state generally forms one or more four-dimensional non-reduced supersymmetry multiplets. This is true even if both particles are individually reducedmultiplet, unless $m_{1}+m_{2}=\left|\Delta_{1}+\Delta_{2}\right|$. Thus the two-particle state generallydoes not saturate the Bogomolnyi bound. This is the first case where we can check whether we get contributions of the form (2.13) from four-dimensional representations. We will see that the two-particle contribution is very simple and general, and often not zero, for the case where both particles are part of reduced multiplets. 
Computing the two-particle contribution is easy if one knows the two-particle $S$ matrix; the $S$-matrix encodes the density of states [28]. In a large box, the particles spend a negligible amount of phase space near each other, so the exact details of the interaction are unnecessary. The $S$-matrix allows one to match the free-particle solution of the equation with $x_{1}>>x_{2}$ with the one for $x_{1}<<x_{2}$.

Consider a two-particle state $\left|i\left(p_{1}, p_{2}\right)\right\rangle$ which scatters entirely into another state $\left|j\left(p_{1}^{\prime}, p_{2}^{\prime}\right)\right\rangle$ with $S$-matrix element $S_{i j}\left(p_{1}, p_{2}\right)$. 6 Relativistic invariance ensures that $S_{i j}$ actually depends only on $s \equiv\left(E_{1}+E_{2}\right)^{2}-\left(p_{1}+p_{2}\right)^{2}=m_{1}^{2}+m_{2}^{2}+2 m_{1} m_{2} \cosh \left(\theta_{1}-\theta_{2}\right)$, where we define rapidities via $p=m \sinh \theta$. Generically, $|i\rangle \neq|j\rangle$ for solitons even in elastic forward scattering, where the individual $\Delta$ change. Consider a two-particle wavefunction connecting vacua $a$ and $b$ at the box ends. Properly-matched plane-wave states satisfy

$$
\psi\left(x_{1}, x_{2}\right)= \begin{cases}e^{i p_{1} x_{1}+i p_{2} x_{2}} & x_{1}<x_{2}, \\ e^{i p_{1}^{\prime} x_{1}+i p_{2}^{\prime} x_{2}} S_{i j}\left(\theta_{2}-\theta_{1}\right) & x_{1}>x_{2} .\end{cases}
$$

The momenta can change in the collision; the relations $p_{1}+p_{2}=p_{1}^{\prime}+p_{2}^{\prime}$ and $E_{1}+E_{2}=$ $E_{1}^{\prime}+E_{2}^{\prime}$ give us the final momenta in terms of the initial. Thus we can write $p_{1}^{\prime}=p_{1}^{\prime}\left(p_{1}, p_{2}\right)$.

Since our system is in a box of length $L$, an allowed wavefunction must vanish at the walls. This quantizes the momenta just as in the free case, but here the two quantization relations are coupled. Requiring the wavefunction vanish at $x_{1}=0$ means making a standing wave by subtracting the solution with opposite $p_{1}$. When making it vanish at $x_{1}=L$, we use the second relation in (4.2), and it follows that

$$
e^{i p_{1}^{\prime}\left(p_{1}, p_{2}\right) L} S_{i j}\left(\theta_{2}-\theta_{1}\right)=e^{i p_{1}^{\prime}\left(-p_{1}, p_{2}\right) L} S_{i j}\left(\theta_{2}+\theta_{1}\right)
$$

where we note that $p_{1}^{\prime}\left(-p_{1}, p_{2}\right)$ is not necessarily equal to $-p_{1}^{\prime}\left(p_{1}, p_{2}\right)$. Requiring the vanishing at $x_{2}=0$ and $x_{2}=L$ gives another equation:

$$
e^{-i p_{2}^{\prime}\left(p_{1}, p_{2}\right) L} S_{i j}\left(\theta_{2}-\theta_{1}\right)=e^{-i p_{2}^{\prime}\left(p_{1},-p_{2}\right) L} S_{i j}\left(-\theta_{2}-\theta_{1}\right)
$$

Taking the log of (4.3) gives

$$
2 n \pi=k_{1} L+I m \ln \frac{S_{i j}\left(\theta_{2}-\theta_{1}\right)}{S_{i j}\left(\theta_{2}+\theta_{1}\right)},
$$

6 We neglect processes which take two particles to more than two (which should be a good assumption in the infrared limit).

7 We also need to define the states so that when $\left|i\left(p_{1}, p_{2}\right)\right\rangle$ scatters only into $\left|j\left(p_{1}^{\prime}, p_{2}^{\prime}\right)\right\rangle$, then $\left|j\left(p_{1}^{\prime}\left(-p_{1}, p_{2}\right), p_{2}^{\prime}\left(-p_{1}, p_{2}\right)\right)\right\rangle$ also scatters only into $\left|i\left(-p_{1}, p_{2}\right)\right\rangle$. In other words, the scattering remains diagonal even after a particle bounces off the wall. 
where $n$ is an integer, and we define the kinematic factors

$$
k_{1}=p_{1}^{\prime}\left(p_{1}, p_{2}\right)-p_{1}^{\prime}\left(-p_{1}, p_{2}\right) \quad k_{2}=p_{2}^{\prime}\left(p_{1}, p_{2}\right)-p_{2}^{\prime}\left(p_{1},-p_{2}\right)
$$

(notice that for forward elastic scattering, $k_{i}=2 p_{i}$ ). Taking the log of (4.4) gives another relation:

$$
2 \bar{n} \pi=k_{2} L-I m \ln \frac{S_{i j}\left(\theta_{2}-\theta_{1}\right)}{S_{i j}\left(-\theta_{2}-\theta_{1}\right)},
$$

The contribution to the index from the two-particle state $i$ comes from summing over all integers $n$ and $\bar{n}$, so that $p_{1}$ and $p_{2}$ are greater than zero. Since we have free on-shell states, the energy is just the free-particle energy. The levels are close together because the box is large, so we replace these sums with integrals. We also make the integral over $\theta_{1}$ and $\theta_{2}$, so that we must multiply by the density of states $g_{f_{i}}$, which is the Jacobian

$$
g_{f_{i}}=\frac{\partial n}{\partial \theta_{1}} \frac{\partial \bar{n}}{\partial \theta_{2}}-\frac{\partial n}{\partial \theta_{2}} \frac{\partial \bar{n}}{\partial \theta_{1}} .
$$

The relations (4.5) and (4.6) give $\partial n / \partial \theta_{i}$ and $\partial \bar{n} / \partial \theta_{i}$ each as the sum of two terms, one proportional to $L$ (the "free" piece) and the other involving the $S$-matrix. Thus $g_{f_{i}}$ has a piece proportional to $L^{2}$. This results in the two-free-particle contribution to the index. However, summing over each four-dimensional representation gives a contribution of the form (2.13), and the $L^{2}$ piece vanishes in $g_{f+2}-g_{f}$. We know this must happen, from the discussion following (2.9). The contribution proportional to $L$ from a state with fermion number $f_{i}$ is

$$
\begin{aligned}
f_{i} e^{i \pi f_{i}} \frac{L}{2(2 \pi)^{2}} \iint d \theta_{1} d \theta_{2} & \left(\left(\frac{\partial}{\partial \theta_{1}}+\frac{\partial}{\partial \theta_{2}}\right)\left(k_{1}+k_{2}\right)\right) \\
& \frac{\partial}{\partial \theta_{1}} \operatorname{Im} \ln S_{i j}\left(\theta_{2}-\theta_{1}\right) e^{-\beta\left(m_{1} \cosh \theta_{1}+m_{2} \cosh \theta_{2}\right)} .
\end{aligned}
$$

To simplify the expression, we have rewritten the rapidity integrals to go from $-\infty$ to $\infty$ by using the fact that $S(-\theta)=S^{*}(\theta)=1 / S(\theta)$, which follows from analyticity and unitarity of the $S$-matrix.

We now specialize to the case where both particles are in reduced multiplets $\mathrm{B}$. The result simplifies remarkably, and only depends on the individual $\Delta$ 's of the particles and not on any details of the $S$-matrix. We decompose the initial states into four-dimensional

8 It is perfectly conceivable that only configurations comprised of particles belonging to reduced multiplets contribute to the index. 
supersymmetry representations $i$ with fermion numbers $\left(f_{i}, f_{i}+1, f_{i}+1, f_{i}+2\right)$ as in (2.10). Denoting a reduced multiplet by $(d, u)$, a two-particle state with fermion number $f_{i}+2$ is of the form $\left|u_{1} u_{2}\right\rangle_{i}$, while the one with fermion number $f_{i}$ is $\left|d_{1} d_{2}\right\rangle_{i}$. By fermionnumber conservation and supersymmetry, $\left|u_{1} u_{2}\right\rangle_{i}$ must scatter only into a state $\left|u_{1}^{\prime} u_{2}^{\prime}\right\rangle_{j}$ and likewise $\left|d_{1} d_{2}\right\rangle_{i}$ must scatter only into a state $\left|d_{1}^{\prime} d_{2}^{\prime}\right\rangle_{j}$. We denote the corresponding $S$-matrix elements by $a_{i j}$ and $\tilde{a}_{i j}$ respectively.

The relation (2.13) means that we do not need to calculate all of the densities: we only need the difference $g_{f_{i}+2}-g_{f_{i}}$. Looking at (4.8), we see that the index thus depends only on the ratio of $S$-matrix elements $a_{i j} / \tilde{a}_{i j}$. The striking fact is that this ratio can be found without knowing the full $S$-matrix; it follows from the supersymmetry alone. We know that $Q^{+} \bar{Q}^{-}\left|f_{i}\right\rangle=\lambda_{i}\left|(f+2)_{i}\right\rangle$, where $\lambda_{i}$ depends on the details of the representation $i$. The $S$-matrix commutes with the supersymmetry generators, which means that the diagram

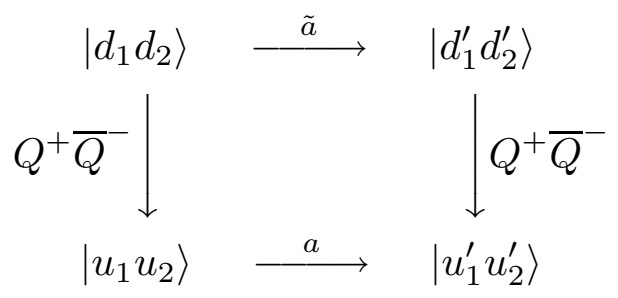

must commute. This implies that

$$
\frac{a_{i j}}{\tilde{a}_{i j}}=\frac{\lambda_{j}}{\lambda_{i}} .
$$

We can find the $\lambda_{i}$ from one-particle information. The supersymmetry is represented on a doublet with $m=|\Delta|$ as

$$
\begin{aligned}
Q^{-}|u(\theta)\rangle & =\sqrt{m} e^{\theta / 2}|d(\theta)\rangle & \bar{Q}^{+}|u(\theta)\rangle & =\omega \sqrt{m} e^{-\theta / 2}|d(\theta)\rangle \\
Q^{+}|d(\theta)\rangle & =\sqrt{m} e^{\theta / 2}|u(\theta)\rangle & \bar{Q}^{-}|d(\theta)\rangle & =\omega^{*} \sqrt{m} e^{-\theta / 2}|u(\theta)\rangle,
\end{aligned}
$$

where $\omega=\Delta /|\Delta|$. All other actions annihilate the states. The supersymmetry is defined on multi-particle states in the usual manner. Since $Q$ is fermionic, one picks up phases when $Q$ is brought through a particle with fermion number. For example, bringing $Q$ through a fermion results in a minus sign. Since we have fractional charges, we must generalize this notion, so that the action of $Q^{ \pm}$on the tensor product of two states is

$$
Q^{ \pm} \otimes 1+e^{ \pm i \pi F} \otimes Q^{ \pm}
$$


The charges $\bar{Q}^{\mp}$ act with the same phases as $Q^{ \pm}$. In our two-particle case of interest, we have

$$
\begin{aligned}
Q^{+} \bar{Q}^{-} & \left|d_{1}\left(\theta_{1}\right) d_{2}\left(\theta_{2}\right)\right\rangle \\
& =e^{i \pi f_{1}} \sqrt{m_{1} m_{2}}\left(\omega_{1}^{*} e^{\left(\theta_{2}-\theta_{1}\right) / 2}-\omega_{2}^{*} e^{\left(\theta_{1}-\theta_{2}\right) / 2}\right)\left|u_{1}\left(\theta_{1}\right) u_{2}\left(\theta_{2}\right)\right\rangle .
\end{aligned}
$$

The quantity of relevance in (4.8) is thus

$$
\frac{\partial}{\partial \theta_{1}} \ln \frac{a_{i j}}{\tilde{a}_{i j}}=\frac{\partial}{\partial \theta_{1}} \ln \frac{\sinh \left(\frac{\theta_{2}-\theta_{1}}{2}+\mu\right)}{\sinh \left(\frac{\theta_{2}^{\prime}-\theta_{1}^{\prime}}{2}+\mu^{\prime}\right)}
$$

where $\mu=\frac{1}{2} \ln \omega_{2} \omega_{1}^{*}$. The contribution of two reduced multiplets to the index $Q$ thus depends only on $\Delta_{1}, \Delta_{2}, \Delta_{1}^{\prime}$ and $\Delta_{2}^{\prime}$ (the masses and hence the $\theta^{\prime}$ follow from this because $m=|\Delta|)$, and is

$$
\begin{aligned}
e^{i \pi f_{i}} \frac{i \beta}{2(2 \pi)^{2}} \iint d \theta_{1} d \theta_{2} & \left(\left(\frac{\partial}{\partial \theta_{1}}+\frac{\partial}{\partial \theta_{2}}\right)\left(k_{1}+k_{2}\right)\right) \\
\frac{\partial}{\partial \theta_{1}} & I m \ln \frac{\sinh \left(\frac{\theta_{2}-\theta_{1}}{2}+\mu\right)}{\sinh \left(\frac{\theta_{2}^{\prime}-\theta_{1}^{\prime}}{2}+\mu^{\prime}\right)} e^{-\beta\left(m_{1} \cosh \theta_{1}+m_{2} \cosh \theta_{2}\right)}
\end{aligned}
$$

The result simplifies in the case of elastic scattering, where the masses of the particles do not change. (Forward elastic scattering is the only allowed process in integrable theories.) For forward elastic scattering, the kinematic prefactor in (4.14) is $2 m_{1} \cosh \theta_{1}+2 m_{2} \cosh \theta_{2}$. Moreover, for forward or backward elastic scattering, one has

$$
\theta_{1}-\theta_{2}=\theta_{1}^{\prime}-\theta_{2}^{\prime}, \quad \mu^{\prime}=-\mu
$$

showing clearly that the elastic two-particle contribution to the index vanishes only when $\mu=0, i \pi / 2$.

For the two-particle contribution from all the reduced multiplets, we sum (4.14) over all pairs. We have thus seen that two-kink contribution to the index from kinks belonging to reduced supersymmetry multiplets is non-vanishing and easily computable.

The results in this section can be compared with the infra-red limit of the index as obtained from the $t t^{*}$ differential equations discussed in the previous section. It is non-trivial that they agree, but they must. This is being investigated numerically in some examples of $N=2$ theories which are not integrable [25]. In the next sections we will focus on integrable theories since then we can obtain, via the exact $S$ matrix and thermodynamics, exact integral equations for the index which can be compared with the $t t^{*}$ equations along the entire renormalization group flow. 


\section{5. $\operatorname{Tr} F(-1)^{F} e^{-\beta H}$ in integrable theories}

In this section we will show how to compute the index for an integrable theory, using the exact $S$-matrix. Integrable theories in two dimensions have been under intensive investigation recently. These theories are characterized by the existence of infinitely many conserved charges, which essentially allows one to solve these theories explicitly. In particular the scatterings are purely elastic; the particles behave as if they are free particles and as they pass through each other they just pick up phases (modulo change of internal indices). The multi-particle $S$-matrix factorizes into two-body $S$-matrices and these are often completely determined by the symmetries of the theory (plus some minimality assumptions which can be verified [10]). The factorizability and elasticity of the $S$-matrix in an integrable theory implies that we can assign rapidities (momenta) to individual particles even in multi-particle configurations. In particular the total energy of multi-particle configurations is simply the sum of the individual ones. The only effect of the interaction is to shift the density of allowed states. This is an ideal situation for computation of our index; the non-trivial part of our index (2.13) results precisely from a discrepancy between densities of states within a non-reduced supersymmetry multiplet.

It is clear that we can in principle continue the analysis of the previous section, using the exact $S$-matrix of an integrable theory, to calculate higher-order corrections in the infra-red expansion. In fact, we can do much better. There is a trick (known as the Thermodynamic Bethe Ansatz [10]) which allows us to compute the index exactly along the entire renormalization group flow, even in the ultra-violet limit! The idea is to not fix the number of solitons, but to consider a thermodynamic ensemble of them. We then minimize the free energy in the ensemble. As we will review, this allows us to calculate exactly, i.e. non-perturbatively, $\operatorname{Tr} F(-1)^{F} e^{-\beta H}$ in an integrable theory with a known exact $S$-matrix. In fact it is no more difficult to compute the more general quantity $\log \operatorname{Tr}\left(e^{i \alpha F} e^{-\beta H}\right)$. This allows us to test our claim that while this quantity depends on the $D$-terms its first derivative with respect to $\alpha$ at $\alpha=\pi$ is independent of the $D$-terms. Even more generally, let us consider the free energy $\mathcal{F}_{\mu_{a}}(\beta)$ with chemical potentials $\mu_{a}$ for the various conserved species labels

$$
-\beta \mathcal{F}_{\mu_{a}}(\beta)=\ln \operatorname{Tr}\left(e^{\beta \sum_{a} \mu_{a} N_{a}} e^{-\beta H}\right)
$$

where $N_{a}$ is the number operator for conserved species $a$. Using the exact $S$-matrix we can obtain an exact expression for $\beta \mathcal{F}_{\mu_{a}}(\beta)$ by finding the minimum value of 
$\beta E-S-\beta \sum_{a} \mu_{a} N_{a}$ in the space of all states, where $S$ is the entropy. Choosing the chemical potentials in (5.1) such that $\beta \sum_{a} \mu_{a} N_{a}=i \alpha F$, we thereby obtain $\operatorname{Tr}\left(e^{i \alpha F} e^{-\beta H}\right)$.

Since the $S$-matrix of an integrable theory preserves rapidities and some set of species labels $a$, a general, multi-soliton state can be characterized by a collection of distributions $\rho_{a}(\theta)$ of rapidities occupied by the various solitons in the multi-soliton state. In particular, the energy of this state is given by

$$
E=\sum_{a} \int d \theta \rho_{a}(\theta) m_{a} \cosh \theta
$$

To do thermodynamics we need to calculate the entropy $S$ and so we need to know the distributions $P_{a}(\theta)$ of available levels as well as the above distributions $\rho_{a}(\theta)$ of occupied levels. In particular

$$
S=\sum_{a} \int d \theta P_{a} \log P_{a}-\rho_{a} \log \rho_{a}-\left(P_{a}-\rho_{a}\right) \log \left(P_{a}-\rho_{a}\right),
$$

corresponding to one particle allowed per level.

Using the exact, factorizable $S$-matrix it can be found that the distributions of available levels are given in terms of the distributions of occupied levels in the general manner:

$$
2 \pi P_{a}(\theta)=m_{a} L \cosh \theta+\sum_{b} \int d \theta^{\prime} \rho_{b}\left(\theta^{\prime}\right) \phi_{a b}\left(\theta-\theta^{\prime}\right)
$$

The $m_{a} L \cosh \theta$ term in (5.4) is the usual density of available states for a free particle, the $\phi_{a b}$ reflect the interaction with the other particles, as given by the exact $S$-matrix. If the $S$-matrix is diagonal, with species $a$ and $b$ scattering with the phase shift $S_{a b}$, the interaction is seen to be given by $\phi_{a b}(\theta)=-i \partial \log S_{a b}(\theta) / \partial \theta$. For non-diagonal S-matrices such as our $N=2 \mathrm{~S}$-matrices, it is generally difficult to obtain the $\phi_{a b}$ from the $S$-matrix; one needs to find the eigenvalues of the multi-particle transfer matrices.

Now we minimize $\beta E-S-i \beta \sum_{a} \mu_{a} N_{a}$, expressed in terms of the above distributions, with respect to the $\rho_{a}(\theta)$ subject to the constraints (5.4). Defining the quantities $\epsilon_{a}(\theta)$ by

$$
\frac{\rho_{a}(\theta)}{P_{a}(\theta)}=\frac{e^{\beta \mu_{a}-\epsilon_{a}(\theta)}}{1+e^{\beta \mu_{a}-\epsilon_{a}(\theta)}},
$$

it is seen that the free energy is given by

$$
\log \operatorname{Tr}\left(e^{\beta \sum_{a} \mu_{a} N_{a}} e^{-\beta H}\right)=-\sum_{a} m_{a} L \int \frac{d \theta}{2 \pi} \cosh \theta \ln \left(1+e^{\beta \mu_{a}-\epsilon_{a}(\theta)}\right),
$$


where the $\epsilon_{a}(\theta)$ are obtained as the solutions to the coupled integral equations:

$$
\epsilon_{a}(\theta)=m_{a} \beta \cosh (\theta)-\sum_{b} \int \frac{d \theta^{\prime}}{2 \pi} \phi_{a b}\left(\theta-\theta^{\prime}\right) \ln \left(1+e^{\beta \mu_{b}-\epsilon_{b}\left(\theta^{\prime}\right)}\right) .
$$

These are the thermodynamic Bethe ansatz [10] integral equations with chemical potentials 29.9 Our interest is in the case where the chemical potentials are chosen such that $\beta \sum_{a} \mu_{a} N_{a}=i \alpha F$. The expression (5.6) was obtained by summing over all boundary conditions at spatial infinity. The different eigenvalues of our matrix trace can be obtained from this expression by inserting appropriate additional chemical potentials. Examples will be discussed in the following section.

\section{Examples}

A generic $N=2$ theory will not, of course, be integrable. Nevertheless, our index for such a theory can be obtained by solving the differential equations discussed in the $t t^{*}$ section of this paper. We would like, however, to compare the computation of the index from the $t t^{*}$ differential equations with the computation from the thermodynamic integral equations. We will thus restrict our examples to integrable theories for which the exact $S$-matrix is known (or conjectured). Examples of such theories are discussed in [11, 12,.

We will focus on integrable theories with spontaneously broken $\mathbf{Z}_{n}$ symmetry. For every $n$ there are a wide variety of such examples, including perturbations of $N=2$ minimal models and Kazama-Suzuki models, supersymmetric $C P^{n-1}$ sigma models, and $N=2$ affine toda theories. For a given value of $n$, the $t t^{*}$ differential equations and the TBA integral equations for all these $\mathbf{Z}_{n}$ integrable theories are found to be essentially the same, the only variation being in the boundary conditions. We will first consider several examples of $\mathbf{Z}_{2}$ theories, namely ordinary $N=0$ sine-Gordon at the particular coupling where it is $N=2$ supersymmetric, $N=2$ sine-Gordon, $N=2$ minimal models perturbed by the least relevant perturbation, and the supersymmetric $C P^{1}$ sigma model. The indices for all of these theories are obtained from the same differential equation, Painleve III. They span all the possible regular boundary conditions. The TBA integral equations also exhibit this fact in a non-trivial guise. We next discuss the more general $\mathbf{Z}_{n}$-type integrable theories starting with the simplest such theory, the $A_{n} N=2$ minimal model perturbed by the most

9 We note that it is straightforward to rederive the TBA equations with our fixed boundary conditions instead of the usual periodic one. The result is the same. 
relevant operator. We finally discuss how to modify the equations in order to determine the index for the other $\mathbf{Z}_{n}$-type theories.

For the most part, we will consider $N=2$ theories which can be described by a LandauGinzburg action 10. Such an action is of the form [26]

$$
\int K\left(X_{i}, \bar{X}_{i}\right)+\int W\left(X_{i}\right)+\int \bar{W}\left(\bar{X}_{i}\right)
$$

where the superfields $X_{i}$ are chiral (in supersymmetric sense, i.e. annihilated by $D^{+}, \bar{D}^{+}$) fields, $W$ is the superpotential and is integrated over half the superspace, and $K$ gives the kinetic terms (the $D$-term) and is integrated over the full superspace. Using topological techniques one can prove that the chiral ring of this theory is exactly characterized by $W$ [6]. In particular the chiral fields of the theory are all products of the fields $X_{i}$ modulo setting to zero $\partial_{i} W$. The chiral ring structure constants entering in (3.3) are obtained by simply multiplying the various products of $X_{i}$ together and imposing the relations $\partial_{i} W=0$.

The physical potential for the theory is

$$
V=K^{i \bar{\jmath}} \partial_{i} W \partial_{\bar{\jmath}} \bar{W}
$$

where $K^{i \bar{\jmath}}$ is the inverse of the Kahler metric $\partial_{i} \partial_{\bar{\jmath}} K$. The vacua $a$ of the theory are thus in one-to-one correspondence with the critical points of $W$. The kinks $k_{a b}$ are the finite energy solutions to the equations of motion connecting the $a$ and $b$ vacua: $X(\sigma=-\infty)=X^{(a)}$, $X(\sigma=+\infty)=X^{(b)}$ (as discussed in [31], not all such kinks are to be regarded as fundamental solitons). The central term in the $N=2$ algebra (2.2) is given simply in terms of the superpotential by $\Delta=2 \Delta W \equiv 2[W(X(\sigma=+\infty))-W(X(\sigma=-\infty))]$. The mass of the $(u, d)$ soliton doublet representation is thus given simply in terms of the superpotential by $m=2|\Delta W|$.

The fractional fermion number in the soliton sector is also given simply in terms of the superpotential by a spectral flow argument [12] or by adiabatic or index theorem techniques 20,21 . The result is that the $(u, d)$ soliton doublet has the fermion numbers $(f, f-1)$ where

$$
f=-\left.\frac{1}{2 \pi}\left(\operatorname{Im} \ln \operatorname{det}\left(\partial_{i} \partial_{j} W(X)\right)\right)\right|_{\sigma=-\infty} ^{\sigma=+\infty}
$$

In all of the integrable theories we consider, the entire spectrum consists of such soliton doublets saturating the bound. There are other integrable $N=2$ theories, for example the theories with superpotential $W=x^{n+1} /(n+1)-\lambda x^{2}$ [31,32, for which this is not the case; exact $S$-matrices for these and many other integrable $N=2$ theories have been recently discussed in [33].

10 The existence of Landau-Ginzburg description seems to apply also to non-supersymmetric and $N=1$ supersymmetric theories [30]. 


\section{1. $N=0$ sine-Gordon at the $N=2$ point}

Ordinary $N=0$ sine-Gordon theory is $N=2$ supersymmetric at a particular coupling [34]. In a manifestly $N=2$ supersymmetric setup, this point is described by a LandauGinzburg superpotential

$$
W=\lambda\left(\frac{X^{3}}{3}-X\right)
$$

with some suitable choice of $K[26]$. The vacua $|a\rangle$ are at $X= \pm 1$. Our matrix index $Q$ has eigenvalues $Q(z)$ and $-Q(z)$, where $z=m \beta$ and $m=2|W(X=1)-W(X=-1)|=\frac{8 \lambda}{3}$. We will first use the $t t^{*}$ equations to find an exact expression for our index in terms of a famous differential equation.

The $t t^{*}$ analysis of this theory was discussed at length in [3]. The result is that the metric on the space of ground states, in the basis spanned by $1, X$, is given by $g=e^{\sigma_{3} u(z) / 2}$. Using (3.3) with the chiral ring $X^{2}=1$, it follows that $u(z)$ satisfies the radial sinh-Gordon equation

$$
\frac{d^{2} u}{d z^{2}}+\frac{1}{z} \frac{d u}{d z}=\sinh u
$$

The radial sinh-Gordon equation is a special case of Painleve III. From relation (3.8) it follows that the index $Q(z)$ is given by

$$
Q(z)=\frac{1}{2} z \frac{d}{d z} u(z)
$$

If we wish, we could eliminate $u(z)$ from these equations and write a differential equation for $Q(z)$

$$
Q^{\prime \prime}-z^{-1} Q^{\prime}=Q \sqrt{4 z^{-2} Q^{\prime 2}+1}
$$

The solutions $u(z)$ to (6.3) behave for $z \rightarrow 0$, i.e. the ultra-violet or conformal limit, as

$$
\begin{aligned}
u(z ; r) & \sim r \log \frac{z}{2}+s+O\left(z^{2-|r|}\right) \quad \text { with }|r|<2 \\
& \sim \pm \log \frac{z}{2} \pm \log \left[-\left(\log \left(\frac{z}{4}+C\right)\right]+O\left(z^{4} \log ^{2} z\right) \quad \text { for }|r|=2\right.
\end{aligned}
$$

where

$$
e^{s / 2}=\frac{\Gamma\left(\frac{1}{2}-\frac{r}{4}\right)}{2^{r} \Gamma\left(\frac{1}{2}+\frac{r}{4}\right)}
$$

and where $C$ is the Euler constant, and $r$ is just a parameter to label the boundary condition. For our theory regularity requires $r=2 / 3$ [3]. From (6.6) it follows that $\pm Q(z=0)= \pm 1 / 3$. This is to be expected, since in this limit the eigenvalues of the $Q$ matrix index are the left plus the right $U(1)$ charge of the Ramond ground states. 
We will now obtain integral equations for the function $Q(z)$ [11], using the exact $S$-matrix obtained in [9,35]. The one particle spectrum of this theory consists of a soliton reduced multiplet $(u, d)$, with mass $m=2|\Delta W|$, and fermion numbers obtained from (6.1) to be $(1 / 2,-1 / 2)$. The soliton connects either vacuum with the other one. A multi-soliton state can be characterized by a distribution $\rho_{1}(\theta)$ of rapidities occupied by the solitons. Because the $u$ and $d$ solitons do not scatter diagonally, we can not assign individual distributions for $u$ type and $d$ type solitons. Instead, there are two additional distributions $\rho_{l}(\theta), l=0, \overline{0}$, which encode the way in which the solitons are distributed as $u$ or $d$ solitons. The distributions $\rho_{l}(\theta)$ arose in [11] in obtaining the eigenvectors and eigenvalues of the multi-particle transfer matrices. We correspondingly have distributions $P_{1}(\theta)$ and $P_{l}(\theta)$ for the density of states. As discussed in [1], these densities satisfy relations of the type (5.4), described by the diagram

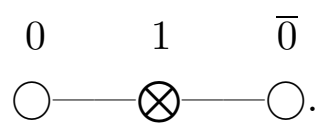

The nodes in this diagram correspond, as labeled, to the species in (5.4). The nodes for species 0 and $\overline{0}$ are open to signify that these species have $m_{a}=0$ in the equations (5.4) (arising from the fact that these species are not physical particles but, rather, account for the additional $u$ and $d$ degree of freedom); the $\otimes$ node has mass $m_{1}=2|\Delta W|$. The functions $\phi_{a b}$ in (5.4) are given by $\phi_{a b}(\theta)=(\cosh \theta)^{-1} l_{a b}$, where $l_{a b}$ is the incidence matrix for the figure, i.e. it is one when species $a$ and $b$ are connected by a line and zero otherwise (and $\left.l_{a a}=0\right)$.

The remaining ingredients required in equation (5.7) are the chemical potentials. These are chosen so that $\beta \sum_{a} \mu_{a} N_{a}=i \alpha F$. Thus we need to express $F$ in terms of the above densities. For a state with a total number $k$ of $u$ solitons and a total number $N-k$ of $d$ solitons, these distributions are defined to satisfy

$$
\int d \theta \rho_{1}(\theta)=N \quad \text { and } \quad \int d \theta\left(P_{0}-\rho_{0}+\rho_{\overline{0}}\right)=k
$$

The fermion number of such a state is $k-(N / 2)$ and so using the above equations along with (5.4) we find

$$
F=\int d \theta\left(\rho_{\overline{0}}(\theta)-\rho_{0}(\theta)\right)
$$

We are ready to use (5.6) and (5.7) to obtain exact integral equations for $\ln \operatorname{Tr} e^{i \alpha F} e^{-\beta H}$. The $m_{a}$ and functions $\phi_{a b}(\theta)$ in these equations are as given above and, 
using (6.7), the chemical potentials should be taken to be $\beta \mu_{1}=0$ and $\beta \mu_{0}=-\beta \mu_{\overline{0}}=i \alpha$. First note that at $\alpha=\pi$ (Witten's index) the equations (5.7) are solved by

$$
e^{-\epsilon_{1}(\theta)}=\epsilon_{0}(\theta)=\epsilon_{\overline{0}}(\theta)=0
$$

for all $\theta$. From (5.6) is is then seen that $L^{-1} \log \operatorname{Tr}(-1)^{F} e^{-\beta H}=0$ when $L \rightarrow \infty$, as expected.

To move slightly away from Witten's index, take $\alpha=\pi+h$ with $h$ small and keep terms only up to $O(h)$. The equations (5.7) are then solved by $e^{-\epsilon_{1}(\theta)}=h e^{-A(\theta)}$, and $\epsilon_{0}(\theta)=\epsilon_{\overline{0}}(\theta)=h B(\theta)$, where

$$
\begin{aligned}
& A(\theta ; z)=z \cosh \theta-\int \frac{d \theta^{\prime}}{2 \pi} \frac{1}{\cosh \left(\theta-\theta^{\prime}\right)} \ln \left(1+B^{2}\left(\theta^{\prime} ; z\right)\right) \\
& B(\theta ; z)=-\int \frac{d \theta^{\prime}}{2 \pi} \frac{1}{\cosh \left(\theta-\theta^{\prime}\right)} e^{-A\left(\theta^{\prime} ; z\right)}
\end{aligned}
$$

Using (5.6) and (2.9), the $Q$-matrix eigenvalues are given by $\pm Q(z=m \beta)$ where, in terms of the solution to the above equations

$$
Q(z)=z \int \frac{d \theta}{2 \pi} \cosh \theta e^{-A(\theta ; z)}
$$

We have two exact expressions for the index $Q(z)$, the differential equation (6.3) and (6.4) (or (6.5)) obtained from $t t^{*}$ considerations, and the integral equations (6.8) and (6.9) obtained from $S$-matrix and thermodynamic considerations. These expressions must agree! We know of no way, however, to show directly from the equations that this is the case. Physics has proven a highly non-trivial statement about the above equations. A check is that the ultra-violet limit for the index using the two different equations give exactly the same result $Q(z=0)=1 / 3$. Also, using results from [36] concerning the Painleve III differential equation, it can be seen (after some algebra) that the function $Q(z)$ has an expansion

$$
Q(z)=-z \frac{d}{d z} \sum_{n=0}^{\infty} \frac{2\left(2 \cos \left(\frac{\pi}{2}(2-r)\right)\right)^{2 n+1}}{2 n+1} \int \prod_{i=1}^{2 n+1} \frac{e^{-z \cosh \theta}}{\cosh \left(\frac{\theta_{i}-\theta_{i+1}}{2}\right)} \frac{d \theta_{i}}{4 \pi}
$$

$\left(\theta_{2 n+2} \equiv \theta_{1}\right)$ and, for later use, we have restored the boundary condition parameter $r$ from (6.6); for the present example $r=2 / 3$. 11 This is the type of infra-red (large $z$ ) expansion

11 A similar expression arose in the computation of Ising-model form factors [37. 
which we would expect for our index; the first term is the usual Bessel function. Also, only odd numbers of solitons contribute because only then are the vacua at spatial infinity different. It is easy to see that the two-particle contribution computed in section 4 vanishes, because $\mu=i \pi / 2$ here. By expanding out the integral equations (6.9) and (6.8) it is easily seen that the one-soliton and the three-soliton contribution agree with the $n=0$ and $n=1$ terms in the PIII expression (6.10); after that the comparison becomes more difficult to check directly. We have numerically verified (to real precision) that the function $Q(z)$ obtained from (6.3) and (6.4) does, indeed, agree with that obtained from (6.9) and (6.8). It would be interesting to see how difficult it is to find a direct mathematical argument to verify this. In particular, would we have to re-invent the physics argument, in disguise, to prove their equality?!

\section{2. $N=2$ Super sine-Gordon}

As our next example, we consider the $N=2$ super sine-Gordon theory given by the Lagrangian

$$
\int d^{2} z d^{4} \theta X \bar{X}+\frac{m}{4}\left(\int d^{2} z d^{2} \theta \cos g X+h . c .\right)
$$

The coupling $g$, by a redefinition of $X$ and $\bar{X}$, can be taken to be real. Because our index is independent of the $D$-term, it must, in fact, be independent of the coupling $g$ since, by rescaling the chiral fields, $g$ can be eliminated from the $F$-term and put into the $D$-term. The general quantity $Z(\alpha, \beta)=\operatorname{Tr} e^{i \alpha F} e^{-\beta H}$ can be calculated via the TBA equations since this is an integrable theory. $Z(\alpha, \beta)$ depends on $g$ as a sign of its dependence on the $D$-term. We will show that our index, the first derivative with respect to $\alpha$ at $\alpha=\pi$, is independent of $g$ as expected by our general arguments in sects. 2 and 3 .

The vacua of the $N=2$ sine-Gordon theory are the points $g X_{a}=a \pi$ for $a \in \mathbf{Z}$. We thus have an infinite number of possible vacua for the boundary conditions at $\sigma= \pm \infty$. Because of the symmetry $g X \rightarrow g X+\pi$, a configuration with vacuum $X_{a}$ to the left and vacuum $X_{b}$ to the right is equivalent to one with vacuum $X_{a+n}$ to the left and vacuum $X_{b+n}$ to the right. Consider the contribution $Q_{a b}$ to our index from a fixed boundary condition $(a b)$. Then, this symmetry implies that $Q_{a b}=Q_{a+n, b+n}$. The eigenvalues of a matrix $M_{i j}$ whose entries depend only on $i-j$ are easily found by Fourier transform. The eigenvalues $Q(\Theta)$ are parametrized by an angle $\Theta$ and given by

$$
Q(z ; \Theta)=\sum_{l=-\infty}^{\infty} e^{i \Theta l} Q_{i, i+l}(z)
$$


In other words, we weight configurations by $e^{i \Theta T}$ where $T$ is the topological charge.

Using the results of [3] and (3.8), the index eigenvalues $Q(z ; \Theta)$ are all solutions of the same PIII differential equation (6.3) obtained in the last example, where only the boundary condition (6.6) depends on $\Theta$. Regularity requires [3] the solution $Q(z, \Theta)$ to behave (with $z=m \beta)$ as $(6.6)$ with

$$
r(\Theta)=2\left(1-\frac{2 \Theta}{\pi}\right) \quad \text { for } \quad 0 \leq \Theta \leq \pi
$$

with $r(\Theta)$ defined outside this interval by $r(\Theta+\pi)=-r(\Theta)$, a consequence of the fact that, in (6.12), only configurations with odd $l$ contribute. By varying $\Theta$, we obtain all regular solutions of Painleve III.

Now we come to the analysis of this theory from the viewpoint of the TBA. The close connection between the index in this example and that in the previous example can also be seen from the exact $S$-matrix and associated integral equations. The important point is that every sine-Gordon soliton is the same $(u, d)$ supermultiplet, with fermion numbers $(1 / 2,-1 / 2)$, seen in the previous example. The (conjectured [38]) $S$-matrix for the $N=2$ sine Gordon theory is simply the tensor product of the $S$-matrix for the theory considered in the previous example with the $S$-matrix for the $N=0$ sine-Gordon [9] at coupling $g_{N=0}^{\text {bare }}=g$. The TBA integral equations for this theory are obtained by combining those of the previous section with the TBA system for $N=0$ sine-Gordon[12]. The TBA system of integral equations for $N=0$ sine-Gordon at generic coupling $g$ is of the usual form (5.7) but with an infinite number of species $a$ and a complicated set of $\phi_{a b}(\theta)$. For the sake of brevity we will thus focus on a nice set of couplings, $g^{2}=8 \pi s$ for $s$ a positive integer $s \geq 2$, where the equations simplify [39]. Of course, our index is independent of the coupling $g$ so we can work with any coupling we please. We will verify this fact, though the more general quantity (2.4) does depend on $s$.

At the coupling $g^{2}=8 \pi s$, as discussed in [12], we obtain a TBA system of coupled integral equations of the usual form (5.7) for $s+3$ functions $\epsilon_{a}(\theta)$. The masses $m_{a}$ and $\phi_{a b}(\theta)$ entering in the equations (5.7) for this theory are described by the figure

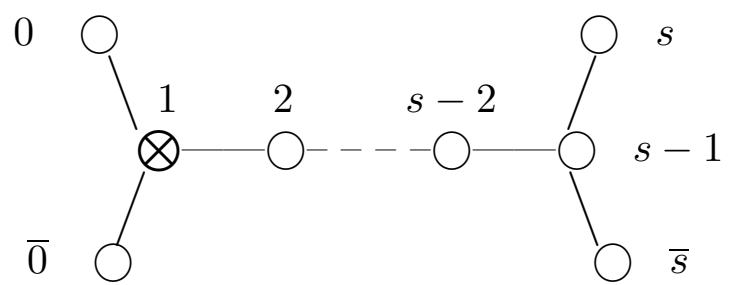


where every node in the diagram corresponds to a species in the equations (5.7). The $\otimes$ node, labelled by 1, corresponds to the soliton; its mass in the equations (5.7) is that of the soliton. The other species have open nodes to signify that they have $m_{a}=0$ in the equations (5.7). Again, the role of these additional species is to account for the additional degrees of freedom (i.e. $u$ or $d$, and which vacua they connect). As in the previous example, the $\phi_{a b}(\theta)=(\cosh \theta)^{-1} l_{a b}$ where $l_{a b}$ is the incidence matrix for the above figure. The massive node and the nodes labelled 0 and $\overline{0}$ come from the $N=2$ part of the $S$-matrix; they correspond precisely to the species in the previous example. The massive node connected to the $s$ open nodes are the TBA species for $N=0$ sine-Gordon at coupling $\left(g_{N=0}^{\text {bare }}\right)^{2}=8 \pi s$. This result is obtained by using a technique known as the algebraic Bethe ansatz to find the eigenvalues of the multi-soliton sine-Gordon transfer matrices (see the appendix of [39]). The TBA system for the tensor-product $S$-matrix is obtained by joining the two component TBA systems at the massive node as described by the above figure.

As in the last example, the fermion number is given by

$$
F=\int d \theta\left(\rho_{\overline{0}}(\theta)-\rho_{0}(\theta)\right)
$$

whereas the sine-Gordon topological charge (number of solitons minus anti -solitons) is given by 39]

$$
T=s \int d \theta\left(\rho_{\bar{s}}(\theta)-\rho_{s}(\theta)\right)
$$

where the species labels are as given in the above figure. By introducing chemical potentials $\beta \mu_{\overline{0}}=-\beta \mu_{0}=i \alpha$ and $\beta \mu_{\bar{s}}=-\beta \mu_{s}=i s \Theta$, with the other chemical potentials zero, the equations (5.7) and (5.6) provide integral equations to compute $\log \operatorname{Tr} e^{i \alpha F} e^{i \Theta T} e^{-\beta H}$ exactly. For generic $\alpha$, the integral equations, in particular the number of functions $\epsilon_{a}(\theta)$, clearly depend on $s$. In the infra-red expansion, one sees that the solutions of these equations are in fact different. Thus $\ln \operatorname{Tr}\left(e^{i \alpha F} e^{i \Theta T} e^{-\beta H}\right)$ depends on the coupling $g^{2}=8 \pi s$, as expected.

At $\alpha=\pi$, the solution of the equations (5.7) described by the above figure with the above chemical potentials is given by $\epsilon_{a}(\theta)$ independent of $\theta: \epsilon_{0}(\theta)=\epsilon_{\overline{0}}(\theta)=e^{-\epsilon_{1}(\theta)}=0$, and

$$
\begin{aligned}
& e^{-\epsilon_{a}(\theta)}=\left(\frac{\sin a \Theta}{\sin \Theta}\right)^{2}-1 \quad \text { for } a=2, \ldots, s-1 \\
& e^{-\epsilon_{s}}=e^{-\epsilon_{\bar{s}}}=\frac{\sin (s-1) \Theta}{\sin \Theta}
\end{aligned}
$$

As expected by supersymmetry, (5.6) gives $L^{-1} \log \operatorname{Tr}(-1)^{F} e^{-\beta H}=0$ in the $\mathrm{E} \rightarrow \infty$ limit. 
We now move slightly away from Witten's index. At $\alpha=\pi+h$ with $h$ small, the solution of the equations (5.7) is given by $\epsilon_{0}(\theta)=\epsilon_{\overline{0}}(\theta)=h B(\theta)$, and $e^{-\epsilon_{1}(\theta)}=h A(\theta)$, where the functions $A(\theta)$ and $B(\theta)$ obey the equations

$$
\begin{aligned}
& A(\theta)=z \cosh \theta-\ln (2 \cos \Theta)-\int \frac{d \theta^{\prime}}{2 \pi} \frac{1}{\cosh \left(\theta-\theta^{\prime}\right)} \ln \left(1+B^{2}\left(\theta^{\prime}\right)\right) \\
& B(\theta)=-\int \frac{d \theta^{\prime}}{2 \pi} \frac{1}{\cosh \left(\theta-\theta^{\prime}\right)} e^{-A\left(\theta^{\prime}\right)} .
\end{aligned}
$$

The other $\epsilon_{a}(\theta)$ are (to lowest order in $h$ ) still given by the constants (6.15). Using (5.6) and (2.9) we thus see that the $Q$ matrix eigenvalues are given by $Q(z=m \beta ; \Theta)$ where

$$
Q(z ; \Theta)=z \int \frac{d \theta}{2 \pi} \cosh \theta e^{-A(\theta ; z ; \Theta)},
$$

with $A(\theta ; z ; \Theta)$ obtained by solving $(6.16)$.

As expected, our index is independent of the coupling $g$ (i.e. $s$ ). One can check by for example studying the IR expansion of the solutions to the full TBA equations that all the other quantities $I_{l}$ for $l>1 d o$ depend on $g$ and are thus dependent on $D$-terms, in accord with the arguments of section 2. In fact, the entire $N=0$ part of the theory has dropped out of the integral equations, leaving just the constant $\ln 2 \cos \Theta$ in (6.16), which resulted from the constants (6.15). This constant piece is the only reflection of the $N=0$ sine-Gordon structure. The close connections between these integral equations and those computed in the previous example was also to be expected from the $t t^{*}$ considerations; the $\ln (2 \cos \Theta)$ term in (6.16) specifies the boundary conditions in the Painleve III equation. Again, though (6.16) and (6.17) have no obvious connection to the radial sinh-Gordon (or Painleve III) differential equation (6.3) and (6.4), physics proves that the regular solutions are the same.

It is easily seen that (6.16) and (6.17) lead to an expansion of the form

$$
Q(z ; \Theta)=\sum_{n=0}^{\infty}(2 \cos \Theta)^{2 n+1} A_{2 n+1}(z) .
$$

$A_{2 n+1}$ is the contribution from the $(2 n+1)$-soliton sector, and is independent of $\Theta .12$ In the large $m \beta$ limit, $A_{2 n+1}$ is $\left.O\left(e^{-(2 n+1) m \beta}\right)\right)$. We can compare this result with (6.10). We

12 This leads to an amusing intuition for our index. Write this result as

$$
Q(z ; \Theta)=\sum_{n=0}^{\infty} A_{2 n+1}(z)\left(e^{i \Theta}+e^{-i \Theta}\right)^{2 n+1},
$$


immediately arrive at the expression (6.13) relating the boundary condition $r$ on the PIII differential equation to our parameter $\Theta$. This is a further confirmation that the TBA solution is related to PIII solution, as in both cases the dependence on PIII boundary conditions $r$ or equivalently $\Theta$ has the same structure. As a further check, the UV limit $z \rightarrow 0$ of (6.17) and (6.16) is obtained (using results from [40] for taking the UV limit of TBA systems with imaginary chemical potentials) to be

$$
Q(z=0 ; \Theta)=\left(1-\frac{2 \Theta}{\pi}\right)
$$

for $0<\Theta<\pi$, in agreement with (6.3) and (6.4) with the boundary condition specified by (6.6) and (6.13).

At $\Theta=0 \bmod \pi$ we have to be more careful in evaluating the UV limit of the equations (6.17) and (6.16). Exactly as in [41], there is a log piece in the ultra-violet limit. This log piece agrees with the expression (6.6) for the Painleve III solution at $r= \pm 2$.

\section{3. $N=2$ minimal models with least-relevant perturbation}

An $N=2$ minimal model remains integrable when perturbed by its least-relevant operator [32,42]. The effective Landau-Ginzburg superpotential for the perturbed theory is identified to be a Chebyshev polynomial (as conjectured in [3] and confirmed in [11]); e.g. for the perturbed $A_{k+1}$ theory the superpotential is $W_{k}(X=2 \cos \theta)=(2 / k+2) \cos (k+2) \theta$, expressed as a polynomial in $X$. This is the least relevant perturbation of the conformal field theory $W=X^{k+2} / k+2$ in the flat direction of [23]. The chiral ring of this theory yields the $S U(2)_{k}$ fusion rules [43].

The $t t^{*}$ computation of our index here is a simple application of the results from the previous example. The reason is that if we change variables $(k+2) \theta \rightarrow Y$, the above Chebyshev polynomial becomes the superpotential for $N=2$ sine-Gordon. The eigenvalues of the index are thus obtained to be

$$
Q\left(z=m \beta ; \Theta=\frac{\pi n}{k+2}\right),
$$

and compare with (6.12). The factor $e^{i \Theta}$ counts the solitons connecting vacuum $i$ to vacuum $i+1$ (topological charge 1) and the factor $e^{-i \Theta}$ counts anti-solitons connecting vacuum $i$ to vacuum $i-1$ (topological charge -1 ). The fact that they are all weighted by the same factor means that we can obtain the index by weighting each $2 n+1$-soliton configuration by $A_{2 n+1}$. This intuition only applies to the computation of our index, and not to the other thermodynamic quantities. 
for $n=1, \ldots, k+1$, where $Q(z ; \Theta)$ is the function discussed in the previous example.

We now discuss the TBA calculation of the index using the exact $S$-matrix for these theories. The vacua of the $W_{k}(X)$ Chebyshev theory are at $X^{(n)}=2 \cos (\pi n / k+2)$, for $n=1, \ldots, k+1$. The solitons are $N=2$ Bogomolnyi doublets $\left(u_{j}, d_{j}\right)$ connecting vacuum $X^{(j)}$ with vacuum $X^{(j+1)}$, for $j=1, \ldots k$, each identical to that of the example in sect 6.1 ; they all have the same mass and fermion numbers $\left(\frac{1}{2},-\frac{1}{2}\right)$. The structure of $k+1$ vacua on a line is that of the $k$-th RSOS theory [44], which describes the $N=0$ minimal models with least-relevant perturbation. The $N=2$ Chebyshev $S$-matrix is a direct product of this $N=0$ RSOS $S$-matrix with the $N=2 S$-matrix discussed in sect. 6.1 , just as the $N=2$ sine-Gordon $S$-matrix of the previous subsection was the tensor product of the $N=0$ sine-Gordon $S$-matrix with the $N=2 S$-matrix of sect. 6.1 .

There is a well-known reduction from $N=0$ sine-Gordon at a particular coupling to the $k$-th RSOS theory. This same reduction can be used to obtain our $N=2$ Chebyshev theories from the $N=2$ sine-Gordon theory; the common $N=2$ structure just goes along for the ride. We start with the $N=2$ sine-Gordon TBA equations appropriate for $N=2$ sine-Gordon coupling $g^{2}=8 \pi(k+2)$. The equations are described by the diagram of the previous subsection, with $s=k+2$. The reduction of this to our Chebyshev theory requires taking $e^{\beta \mu_{s}}=e^{\beta \mu_{s}}=-1$ [39]. This reduction simply eliminates the nodes labelled $s, \bar{s}$, and $s-1$ from the diagram in the previous subsection, leaving the TBA system discussed in [1].

The solution for this TBA system is the sine-Gordon solution $Q(z ; \Theta=\pi /(k+2))$, giving the largest eigenvalue of the $Q$-matrix for the Chebyshev theory. The remaining eigenvalues of the matrix index are given by $Q(z, \Theta=\pi n /(k+2))$, for $n=1, \ldots, k+1$, and can be seen as other branches of this solution. 13 It follows from (6.17) that the non-zero eigenvalues come in pairs of opposite sign, as they should. As another check, note that in the ultra-violet limit we obtain (6.19) $Q(z=0, \Theta=\pi n /(k+2))=1-(2 n / k+2)$; these are the correct expressions for the left plus right chiral $U(1)$ charges for the Ramond ground states of the conformal theory obtained in the ultra-violet limit.

13 The resulting factors of $2 \cos n \pi /(k+2) \equiv \lambda_{n}$ in (6.18) are the eigenvalues of the RSOS soliton incidence matrix. The number of $N$-soliton configurations can be expressed (for any boundary condition) as $\sum_{n} c_{n} \lambda_{n}^{N}$ with $N$ independent $c_{n}$. This is in accordance with the intuition of the footnote following (6.18). 


\subsection{Supersymmetric $C P^{1}$ sigma model}

The supersymmetric sigma model on $C P^{1}=S^{2}$ is a massive $N=2$ theory. In principle we can consider an arbitrary kahler metric on $C P^{1}$. Varying the metric without changing its kahler class (i.e., preserving the area) is a $D$-term perturbation of the theory and therefore the index only depends on the area. Letting $X$ denote the Kahler form of $C P^{1}$, the chiral ring, which is to be identified as an instanton-modified cohomology ring, is $X^{2}=e^{-A}$ where $A$ is the area of $C P^{1}$ (the two-sphere) [45]. The $t t^{*}$ considerations have been applied to this and $C P^{n-1}$ examples in [46]: using the above ring the index is again given by (6.3) where $z=8 \beta e^{-A / 2}$ and, by regularity, the boundary condition (6.6) is determined to be $\Theta=0$, i.e. $r=2$.

For a generic metric on $C P^{1}$, the supersymmetric sigma model is not an integrable theory. However, since the index only depends on the area, for computation of the index we may as well use a convenient choice of the metric. If we choose the constant-curvature metric on $C P^{1}$ then this theory is integrable [47] and so we can compare the $t t^{*}$ analysis with the TBA analysis. As discussed in [12], the TBA system for this theory is obtained by taking the $k \rightarrow \infty$ limit of the Chebyshev TBA system. Our index for the supersymmetric

$C P^{1}$ sigma model is thus given by (6.16) and (6.17) with $\Theta=0$, in agreement with the $t t^{*}$ result, given our equality between the Painleve III differential equations and these integral equations.

\subsection{The Basic $\mathbf{Z}_{n}$-type $N=2$ Integrable Theories}

All of the previous examples displayed a spontaneously broken $\mathbf{Z}_{2}$ symmetry. The $t t^{*}$ equations for the index in all these examples were the same, the only difference being in the boundary conditions. Likewise, the TBA integral equations for the index in all of these examples were the same, the only difference being in the value of $\Theta$ in (6.16). We will now consider $N=2$ integrable theories with a spontaneously broken $\mathbf{Z}_{n}$ symmetry. The basic such theory is the $A_{n} N=2$ minimal conformal field theory perturbed by the mostrelevant supersymmetry preserving operator. It can be described by the Landau-Ginzburg superpotential [26]

$$
W=\lambda\left(\frac{X^{n+1}}{n+1}-X\right)
$$

This theory is integrable [31; in fact, it can be described by an affine Toda theory with an imaginary coupling and a background charge [32. 
The $t t^{*}$ equations for this example were discussed in [3]. Because theory (6.21) has the $\mathbf{Z}_{n}$ symmetry $X \rightarrow e^{2 \pi i / n} X$, the Ramond ground state metric $g$ is diagonal in the chiral ring basis spanned by $1, X, \ldots, X^{n-1}$. Denoting these diagonal elements by $e^{q_{p}}$, equation (3.3) with the chiral ring relation $X^{n}=1$ yields the following relations for the eigenvalues $Q(z ; p)$ of our matrix index:

$$
Q(z ; p)=z \frac{d}{d z} q_{p}(z) \quad \text { where } \frac{d^{2} q_{p}}{d z^{2}}+\frac{1}{z} \frac{d q_{p}}{d z}+e^{q_{p+1}-q_{p}}-e^{q_{p}-q_{p-1}}=0,
$$

for $p=0, \ldots, n-1$, with $q_{p+n} \equiv q_{p}$ and with the constraint $q_{n-p}=-q_{p}$. For $n=2$, (6.22) reduces to the sinh-Gordon (6.3) and (6.4) of our previous examples. The $\hat{A}_{n-1}$ Toda equations with the constraint $q_{n-p}=-q_{p}$ can be put in the form of $\hat{C}_{m}$ Toda theory for $n=2 m$ or $\hat{B C} C_{m}$ Toda theory for $n=2 m+1$ [3].

Exact integral equations for the above eigenvalues of our matrix index follow from the exact $S$-matrix and TBA analysis discussed in [12]. The vacua of theory (6.21) are at $X^{(j)}=e^{2 \pi i j / n}$. The soliton content consists of $2(n-1)$ solitons forming doublets $\left(u_{r}, d_{r}\right)$ under supersymmetry; the soliton species label $r$ runs from $1, \ldots, n-1$ corresponding to solitons connecting initial and final vacua with $X_{f}=e^{2 \pi i r / n} X_{i}$. The fermion numbers of $\left(u_{r}, d_{r}\right)$ are given by $(r / n, r / n-1)$, and their mass is given by the Bogomolnyi bound $m_{r}=M \sin r \pi / n$ where $M=4 n \lambda /(n+1)$.

The conserved currents require that when a soliton of type $r$, i.e. $u_{r}$ or $d_{r}$, scatters with a soliton of type $s$, the labels $r$ and $s$ scatter diagonally — along with the rapidities. The number $N_{r}$ of solitons of type $r$, i.e. the number of $u_{r}$ solitons plus the number of $d_{r}$ solitons, is thus conserved for $r=1, \ldots, n-1$, as is the total number of $u$ solitons, and the total number of $d$ solitons. A multi- soliton state can thus be characterized by distributions $\rho_{r}(\theta)$ of rapidities occupied by solitons of type $r$, i.e. $u_{r}$ or $d_{r}$, along with, as in the previous examples, two additional distributions $\rho_{l}(\theta)$ and $l=0, \overline{0}$ [12]. The fermion number of the multi-soliton state is again found to be given in terms of the various distributions by (6.7).

Using the exact $S$-matrix it was found in [12] that the distributions $P_{a}(\theta)$ of available levels for the above species $a$ are given in terms of the above occupied distributions $\rho_{a}(\theta)$ by relations of the usual form (5.4) where $m_{r}=M \sin (r \pi / n)$ is the mass of species $r=1, \ldots, n-1, m_{l}=0$ for $l=0, \overline{0}$, and the $\phi_{a b}$ are given by

$$
\begin{aligned}
\phi_{l, l^{\prime}} & =0, \quad \phi_{r, l}(\theta)=\frac{\sin (r \mu)}{\cosh (\theta)-a_{l} \cos (r \mu)}, \\
\phi_{r, s}(\theta) & =\int d t e^{i t \theta}\left(\delta_{r s}-2 \frac{\cosh \mu t \sinh (\pi-r \mu) t \sinh s \mu t}{\sinh \pi t \sinh \mu t}\right),
\end{aligned}
$$


for $r \geq s=1, \ldots n-1$, with $\phi_{a b}=\phi_{b a}$, where $a_{0} \equiv-a_{\overline{0}} \equiv 1$ and $\mu \equiv \pi / n$.

We now calculate the eigenvalues of $\operatorname{Tr}_{a b}\left(e^{i \alpha F} e^{-\beta H}\right)$. The $p$-th eigenvalue of this matrix can be obtained by weighting solitons of type $r$ by $e^{2 \pi i r p / n}$, for $p=$ $0, \ldots, n-1$, and summing over all vacua $(a b)$ at spatial infinity. We will thus calculate $\log \operatorname{Tr} e^{i \alpha F} e^{2 \pi i p r N_{r} / n} e^{-\beta H}$ by using (5.1) with the chemical potentials $\beta \mu_{r}=-2 \pi i r p / n$ and, from (6.7), $\beta \mu_{0}=-\beta \mu_{0}=i \alpha$. Plugging these chemical potentials and the (6.23) into (5.7) and (5.6), we obtain integral equations which determine exactly $\operatorname{Tr}\left(e^{i \alpha F} e^{-\beta H}\right)$.

At $\alpha=\pi$ it is seen by inspection that the solution of the coupled integral equations (5.7) is given by $e^{-\epsilon_{r}(\theta)}=\epsilon_{l}(\theta)=0$, and thus $L^{-1} \log \operatorname{Tr}(-1)^{F} e^{-\beta H}=0$ in the large $L$ limit. We now consider $\alpha=\pi+h$ with $h$ small. The solution of (5.7) is of the form $e^{-\epsilon_{r}}=h e^{-2 \pi i r p / n} e^{-A_{r}}$ and $\epsilon_{l}=h B_{l}$, where the $A_{r}$ and $B_{l}$ satisfy the coupled integral equations

$$
\begin{aligned}
& A_{r}(\theta)=m_{r} \beta \cosh \theta+2 i r p \mu-\sum_{l=0, \overline{0}} \int \frac{d \theta^{\prime}}{2 \pi} \frac{\sin (r \mu) \ln \left(i a_{l}+B_{l}\left(\theta^{\prime}\right)\right)}{\cosh \left(\theta-\theta^{\prime}\right)-a_{l} \cos (r \mu)} \\
& B_{l}\left(\theta^{\prime}\right)=-\sum_{r=1}^{n-1} \int \frac{d \theta^{\prime \prime}}{2 \pi} \frac{\sin (r \mu) e^{-A_{r}\left(\theta^{\prime \prime}\right)}}{\cosh \left(\theta^{\prime}-\theta^{\prime \prime}\right)-a_{l} \cos (r \mu)},
\end{aligned}
$$

where, again, $a_{0}=-a_{\overline{0}}=1, m_{r}=M \sin (r \pi / n)$, and $\mu=\pi / n$. Using (2.9) and (5.6) we have the index eigenvalues $Q(z=M \beta ; p)$, for $p=0, \ldots, n-1$, given by

$$
Q(z ; p)=\sum_{r=1}^{n-1} m_{r} \beta \int \frac{d \theta}{2 \pi} \cosh \theta e^{-A_{r}(\theta ; z ; p)} .
$$

It follows from (6.24) that $A_{r}(\theta ; z ; p)^{*}=A_{n-r}(\theta ; z ; p)$ and $B_{0}(\theta ; z ; p)^{*}=B_{\overline{0}}(\theta ; z ; p)$ and, thus, the above eigenvalues $Q(z ; p)$ are all real, as they should be. Furthermore, $e^{-A_{r}(\theta ; z ; p)}=-e^{-A_{n-r}(\theta ; z ; n-1-p)}$ is a consistent solution of (6.24) for all $r=1, \ldots, n-1$ and $p=0, \ldots, n-1$. It follows that $Q(z ; n-1-p)=-Q(z ; p)$; the non-zero eigenvalues come in opposite pairs as they should.

We can compare these exact results (6.25) and (6.24) with the infra-red expansion of sect. 4. Setting $B_{l}\left(\theta^{\prime}\right)=0$ in (6.24), we obtain the first approximation $e^{-A_{r}} \approx$ $i e^{-i \pi r(2 p+1) / n} e^{-m_{r} \beta \cosh \theta}$. Plugging this into (6.25) we obtain the one-soliton sector contribution to the index, in agreement with (4.1). Plugging this first approximation back into (6.24) we obtain the next approximation

$$
\begin{aligned}
e^{-A_{r}} \approx i e^{-i \pi r(2 p+1) / n} e^{-m_{r} \cosh \theta} & \\
& \left(1-\sum_{s} e^{-i \pi s(2 p+1) / n} \int \frac{d \theta^{\prime}}{2 \pi} \phi_{r+s, 0}\left(\theta-\theta^{\prime}\right) e^{-m_{s} \beta \cosh \theta^{\prime}}\right) .
\end{aligned}
$$


Plugging this into (6.25) gives the contribution to the index coming from the two-soliton sector, in agreement with (4.8) and (4.13).

We have again found two different representations of our index: One in terms of solutions to affine Toda equations (6.22) and the other in terms of solutions to coupled integral equations (6.24) and (6.25). It would be interesting to check this equivalence numerically and verify it analytically.

\subsection{Other $\mathbf{Z}_{n}$ Integrable Theories}

There are a variety of other integrable $N=2$ theories with spontaneously-broken $\mathbf{Z}_{n}$ symmetry whose index can be obtained from the equations of the previous subsection. Examples are the affine Toda generalizations of $N=2$ sine-Gordon, integrable perturbations of $N=2$ Kazama-Suzuki theories described by the $S U(n)_{k}$ generalized Chebyshev polynomial superpotentials in $n-1$ variables [43] and the $C P^{n-1}$ sigma models [12]. We first consider the $S U(n)$ affine Toda theories described by the action

$$
\int d^{2} z d^{4} \theta \sum_{j=1}^{n-1} X_{j} \bar{X}_{j}+\frac{M}{4 n}\left(\int d^{2} z d^{2} \theta \sum_{j=1}^{n} e^{i g\left(X_{j}-X_{j-1}\right)}+h . c .\right)
$$

where $X_{0} \equiv X_{n} \equiv 0$. The vacua of this theory form the $n-1$ dimensional weight lattice of $S U(n)$. This theory has $n-1$ topological charges $T_{r}$ and, as in the sine-Gordon case, the eigenvalues of the $Q$ matrix index can be written as

$$
Q\left(z ; \Theta_{1}, \ldots, \Theta_{n-1}\right)=i \beta L^{-1} \operatorname{Tr} F(-1)^{F} e^{i \sum_{r=1}^{n-1} \Theta_{r} T_{r}} e^{-\beta H}
$$

where the trace runs over all boundary conditions.

The $t t^{*}$ analysis has been applied to this example in [3] where the solutions are expressed in terms of solutions to the affine toda equations (6.22) but now with different boundary conditions, which should now depend on $\Theta_{i}$.

The $S$-matrix for the theory $(6.26)$ is (conjectured to be) the tensor product of the $N=2$ theory discussed in the previous subsection with an additional $N=0$ structure with vacua corresponding to the weight lattice of $S U(n)$ : vacua labelled by $S U(n)$ weights $\mu$ and $\nu$ are connected by a soliton doublet of the $r$-th type, with fermion numbers $(r / n, r / n-1)$ and mass $m_{r}=M \sin (r \pi / n)$, provided the representations satisfy $\mu \otimes \Lambda_{r}=\nu \oplus \ldots$ where $\Lambda_{r}$ is the $r$-th fundamental representation of $S U(n)(r=1, \ldots, n-1)$. As far as $N=2$ supersymmetry is concerned, every $r$-type soliton doublet is identical to the $r$-type doublet 
in the basic $\mathbf{Z}_{n}$ theory discussed in the previous section. We will not explicitly carry out the TBA analysis for this theory. Rather, we will use the fact that, as seen in the previous examples, the only effect of the additional $N=0$ structure on our index is to modify the $A_{r}(\theta)$ equations in (6.24) with some $\theta$-independent constants $C_{r}\left(\Theta_{j}\right)$ (generalizing the term $2 \cos \Theta$ in (6.16) for the affine toda case):

$$
Q\left(z ; \Theta_{1}, \ldots, \Theta_{n-1}\right)=\sum_{r=1}^{n-1} m_{r} \beta \int \frac{d \theta}{2 \pi} \cosh \theta e^{-A_{r}(\theta)}
$$

where the $A_{r}(\theta)$ are solutions to the coupled integral equations

$$
\begin{aligned}
A_{r}(\theta) & =m_{r} \beta \cosh \theta-\ln C_{r}\left(\Theta_{j}\right)-\sum_{l=0, \overline{0}} \int \frac{d \theta^{\prime}}{2 \pi} \frac{\sin (r \mu) \ln \left(i a_{l}+B_{l}\left(\theta^{\prime}\right)\right)}{\cosh \left(\theta-\theta^{\prime}\right)-a_{l} \cos (r \mu)} \\
B_{l}\left(\theta^{\prime}\right) & =-\sum_{r=1}^{n-1} \int \frac{d \theta^{\prime \prime}}{2 \pi} \frac{\sin (r \mu) e^{-A_{r}\left(\theta^{\prime \prime}\right)}}{\cosh \left(\theta^{\prime}-\theta^{\prime \prime}\right)-a_{l} \cos (r \mu)} .
\end{aligned}
$$

These equations lead to an expansion of the form

$$
Q\left(z ; \Theta_{j}\right)=\sum_{N_{1}=1}^{\infty} \ldots \sum_{N_{n-1}=1}^{\infty} C_{1}^{N_{1}} \cdots C_{n-1}^{N_{n-1}} A_{N_{1}, \ldots, N_{n-1}}(z)
$$

where $A_{N_{1}, \ldots N_{n-1}}(z)$ is the contribution from the sector with $N_{r}$ solitons of type $r$ for $r=1, \ldots, n-1$. Comparing (6.30) with (6.27) it is seen that the $C_{r}\left(\Theta_{j}\right)$ are given by the character functions

$$
C_{r}\left(\Theta_{j}\right)=\sum_{\lambda \in L\left(\Lambda_{r}\right)} e^{i \lambda \cdot T}
$$

where $L\left(\Lambda_{r}\right)$ are the weights in the $r$-th fundamental representation $\Lambda_{r}$ of $S U(n)$ (the $S U(n)$ representation with $r$ vertical boxes for its Young tableau), for $r=1, \ldots, n-1$, and $T=\sum_{n} \Theta_{n} \alpha_{n}$ where $\alpha_{n}$ are the simple roots of $S U(n)$. The above characters can be written (as in [43]) as

$$
\sum_{l=0}^{n} C_{l}\left(\Theta_{1}, \ldots, \Theta_{n-1}\right) t^{l}=\prod_{j=1}^{n}\left(1+t e^{i\left(\Theta_{j}-\Theta_{j-1}\right)}\right),
$$

where $\Theta_{0} \equiv \Theta_{n} \equiv 0$; expanding the product in $t$ and equating coefficients of $t^{l}$ on both sides yields the above sums over the fully antisymmetric representations. Plugging these $C_{r}\left(\Theta_{i}\right)$ into $(6.29)$ and $(6.28)$ yields the index $Q\left(z ; \Theta_{j}\right)$. It follows from (6.31) that $C_{r}\left(\Theta_{i}\right)^{*}=C_{n-r}\left(\Theta_{i}\right)$ from which it follows from 6.29$)$ that $A_{r}\left(\theta ; z ; \Theta_{i}\right)^{*}=A_{n-r}\left(\theta ; z ; \Theta_{i}\right)$ 
and $B_{0}\left(\theta ; z ; \Theta_{i}\right)^{*}=B_{\overline{0}}\left(\theta ; z ; \Theta_{i}\right)$. It then follows that the index eigenvalues $Q\left(z ; \Theta_{i}\right)$ are real.

The above integral equations must provide the regular solutions of the Toda differential equations (6.22). As in the PIII case, physics has proven a statement for which there is, as of yet, no purely mathematical proof. In particular this gives an $n-1$ parameter family of regular solutions to radial affine Toda equations (6.22). The dependence of the solutions on these parameters $\left(\Theta_{i}\right)$ is again in line with the intuition discussed in the case of PIII (see footnote 12).

We now consider the theory with superpotential given by the $S U(n)_{k}$ Chebyshev polynomial $W_{n+k}\left(X_{1}, \ldots, X_{n-1}\right)$ in $n-1$ variables discussed in [43]; the generating function for these potentials is

$$
\sum_{p} W_{p}\left(X_{1}, \ldots, X_{n-1}\right) t^{p}=-\log \left(1+\sum_{r=1}^{n-1} X_{r}(-t)^{r}+(-t)^{n}\right) .
$$

These theories have been discussed in [43, 3, 48, 49, [12,50] $\left(S p(N)_{k}\right.$ theories, which might also be integrable, have been found in [51,50]). From the $t t^{*}$ analysis it is possible to see that the index is again related to the affine toda equation [3]. From the TBA integral equations the $(n+k-1) ! /(n-1) ! k$ ! eigenvalues of the matrix index for the $S U(n)_{k}$ theory are expected to be obtained from the equations (6.28) and (6.29) by setting $C_{r}\left(\Theta_{i}\right)=X_{r}(\mu)$ where $X_{r}(\mu)$ are the solutions of $d W_{n+k}\left(X_{r}\right)=0$. This is equivalent 43] to setting $C_{r}\left(\Theta_{i}\right)=S_{\Lambda_{r} \mu} / S_{0 \mu}$, where $S_{\mu \nu}$ is the $S U(n)_{k}$ modular transformation matrix, $\Lambda_{r}$ is the $r$-th fundamental representation $S U(n), \mu$ is one of the $(n+k-1) ! /(n-1) ! k$ ! highest weight representations of $S U(n)_{k}$, and 0 is the identity. These integral equations for the index eigenvalues of the $S U(n)_{k}$ Chebyshev theory generalize the $S U(2)_{k}$ results in subsect. 6.3 and the $S U(n)_{1}$ results in sect. 6.5. Finally, we consider the $C P^{n-1}$ sigma model. Here again, the $t t^{*}$ equations give the affine toda equations 46] (with logarithmic boundary conditions). The $S$-matrix for $C P^{n-1}$ sigma model is obtained from the $k \rightarrow \infty$ limit of this $S U(n)_{k}$ Chebyshev theory [12]. Thus, the index for this sigma model is also given by the above integral equations by setting the $\Theta_{r}=0$, i.e. $C_{r}=n ! / r !(n-r) !$, in $(6.29)$.

\section{Conclusions}

We have seen that for two-dimensional $N=2$ supersymmetric theories $\operatorname{Tr}(-1)^{F} F e^{-\beta H}$ is a (matrix) index in a generalized sense, i.e., it is independent of $D$-term perturbations. 
Though the index is not topological, it is determined exactly via non-linear differential equations which are obtained using only topological data, namely the chiral ring. These non-linear differential equations encode the geometry of the vacua of the theory. This allows us to read off, by an IR expansion, the spectrum of Bogomolnyi saturated states of the theory as well as some aspects of their interaction. In case the theory is integrable and the exact $S$-matrix is known, the index can be computed using TBA methods in terms of solutions to coupled integral equations. It is a very non-trivial statement that in these cases the integral equations thus obtained are equivalent to the differential equations characterizing the geometry of vacua.

It is amusing that one can apply $N=2$ formalism to study polymer physics [39]. The index in this context is the partition function of a single polymer wrapped around a cylinder of perimeter $\beta$.

Given the fact that the integral equations which arose for us in the context of the TBA are equivalent to differential equations, it is very natural to ask if this can be done more generally. In other words, is it always possible to relate integral equations arising for integrable theories through the TBA to ordinary differential equations? A first step in this direction may be to try to prove mathematically why in our case the integral equations of the TBA were equivalent to differential equations.

For non-integrable theories the $t t^{*}$ equations can still be used to compute the index. In the infra-red the leading contribution to the index is universal (4.1). However, even though the normalization of this term can be easily deduced from a Hilbert space interpretation, from the viewpoint of solving the $t t^{*}$ equations this is only fixed by requiring the regularity of the solution (even in the UV regime). Therefore, it is a very non-trivial test of these ideas that the normalization coming from solving the $t t^{*}$ equations in the IR agrees with the Hilbert space interpretation. This has recently been confirmed even in a non-integrable case by solving $t t^{*}$ equations numerically [25].

We have seen that the index basically captures the geometry and interaction of kinks interpolating between supersymmetric vacua. It would be interesting to write a general solution (say for Landau-Ginzburg theories) of the $t t^{*}$ equations in terms of these kinks. Such a thing is not unexpected, given the fact that $t t^{*}$ equations depend only on the superpotential (which is equivalent to knowing the kink spectrum and their geometry) and that the equations are integrable as they can be rephrased as flatness conditions even if the underlying quantum field theory is not integrable, a fact which has been recently elaborated upon in [24]. 
Given the power of the new supersymmetric index in encoding exact results, it would be tempting to look for similar objects in other supersymmetric theories. In particular a very similar setup to what we have discussed in this paper appears naturally in the context of four-dimensional $N=2$ Yang-Mills theory. Again this theory is related to a topological theory [52] and the analog of the chiral fields are the two-cycle observables. In particular for the $S U(2)$ gauge theory in the Higgs phase where $S U(2)$ is broken to $U(1)$, all the known particles of the theory such as the massive gauge particles and the monopoles are known to saturate the Bogomolnyi bound [15] very much as our kinks in the two-dimensional theory saturate the Bogomolnyi bound. In this case the natural generalization of our index seems to be $\operatorname{Tr}(-1)^{F} J^{2} e^{-\beta H}$ where $J$ is the generator of $U(2)$ symmetry of $N=2$ theories 53. It would be exciting to see what exact information about the $S$-matrix of these four-dimensional theories are encoded in such an index.

To formulate our index we needed to put the Hilbert space on infinite line to allow for kinks. If we put the Hilbert space on a periodic circle and thus compute the index on the torus we get zero. This can also be seen by CPT invariance. However if we replace $F$ by $F_{L}$, the left-moving fermion number, in the definition of the index, CPT no longer requires it to vanish (as even on the torus $(-1)^{F_{L}}$ is in general not \pm 1 ). This quantity has already appeared in the context of conformal theories where it is related to the moduli dependence of the gauge and gravitational coupling constants [54,55]. This modified index resembles the generalization of Ray-Singer torsion [56] to conformal theories.

We have seen that $N$-kink configurations each contribute to our index through an 'anomaly' resulting from an inequality in the density of states for a supersymmetric multiplet (2.13). Each of these contributions reminds one of (though it is not the same as) the Callias-Bott-Seeley index [14]. It would be very exciting to uncover the meaning of such a 'topological invariant' for each $N$-kink contribution. Our new index, which sums up the contribution of all $N$-kink configurations, would then encode infinitely many topological invariants into a single function!

\section{Acknowledgements}

We would like to thank C.Imbimbo, W. Leaf-Herrmann, A. Lesniewski and E. Witten for helpful discussions. C.V. would also like to thank the ICTP, where part of this work was done, for hospitality. P.F. was supported by DOE grant DEAC02-89ER-40509, K.I. was supported by NSF grant PHY-87-14654 and an NSF graduate fellowship and C.V. was supported by Packard fellowship and NSF grants PHY-87-14654 and PHY-89-57162. 


\section{Appendix A. An Operatorial Proof of the tt* Equations}

In this appendix we show how the ideas of the present paper can be used to give a quick (although less rigorous) proof of the $t t^{*}$ equations of [3].

We begin by rewriting the $t t^{*}$ equations in terms of the $Q$ matrix only. We have seen in the main body of the paper that

$$
Q_{a b}=\frac{i \beta}{L} \operatorname{Tr}_{a b}(-1)^{F} F e^{-\beta H},
$$

where $a, b$ label boundary conditions at spatial infinity associated to some basis $|a\rangle$ of vacua.

In terms of $Q$ the basic $t t^{*}$ equations read

$$
\begin{gathered}
\bar{D}_{\bar{i}} Q=2 \beta^{2}\left[C_{\tau}, \bar{C}_{\bar{i}}\right] \\
D_{i} Q=2 \beta^{2}\left[C_{i}, \bar{C}_{\bar{\tau}}\right] . \\
\frac{1}{2}\left[Q, C_{j}\right]=-C_{j}+D_{j} C_{\tau} \\
\frac{1}{2}\left[Q, \bar{C}_{\bar{j}}\right]=\bar{C}_{\bar{j}}-\bar{D}_{\bar{j}} \bar{C}_{\bar{\tau}} .
\end{gathered}
$$

where $D_{i}\left(\bar{D}_{\bar{i}}\right)$ denotes the metric covariant derivative and $C_{\tau}$ is the matrix representing multiplication by $W$ (in the case of a Landau-Ginzburg theory, which we assume in this section for simplicity) on the vacua $|a\rangle$. One can think of $Q$ in the special parametrization of the couplings of the theory motivated from the renormalization group as $Q=2\left(A_{\tau}-A_{\tau^{*}}\right)$ with $\beta=\exp \left(\tau / 2+\tau^{*} / 2\right)$, from which one can deduce the above equations from the usual $t t^{*}$ form (note that a chiral operator has an explicit $\tau$ dependence given by $e^{\tau} \phi_{i}$ ). The above equations are written so as to make sense in an arbitrary basis (see also [8]). Eq.(A.3) has the following interpretation. For a quasihomogeneous family of superpotentials $C_{\tau}=0$ and (A.3) just states that the marginal chiral (resp. anti-chiral) deformations have $U(1)$ charge +1 (resp. -1 ). The extra term in the RHS measures the 'deviation from marginality'.

On the other hand, (A.3) allows us to write the full metric connection $A_{i}$ in terms of $Q$ and the ring coefficients. Then we can compute its curvature in terms of $Q$. Because of this, (A.2) and (A.3) together with

$$
\begin{aligned}
D_{i} \bar{C}_{\bar{j}} & =\bar{D} \bar{j}_{j} C_{i}=0 \\
D_{i} C_{j} & =D_{j} C_{i},
\end{aligned}
$$


reproduce all the $\mathrm{tt}^{*}$ equations. (In fact, the second line is a consequence of (A.3) together with known properties of the chiral ring). In particular, we get

$$
\begin{aligned}
& {\left[D_{i}, \bar{D}_{\bar{j}}\right] Q=-\beta^{2}\left[\left[C_{i}, \bar{C}_{\bar{j}}\right], Q\right]} \\
& {\left[D_{i}, \bar{D}_{\bar{j}}\right] C_{\tau}=-\beta^{2}\left[\left[C_{i}, \bar{C}_{\bar{j}}\right], C_{\tau}\right]}
\end{aligned}
$$

from which we read the curvature of the metric connection 4 .

One has also the identity ( $\Delta$ is defined in (2.2))

$$
\left[C_{\tau}, \bar{C}_{\bar{j}}\right]_{a b}=\frac{1}{2} \operatorname{Tr}_{a b}(-1)^{F} \Delta \bar{\phi}_{j} e^{-\beta H}
$$

This equation deserves a comment. The simplest way of proving it is to choose the vacuum basis $|a\rangle$ to correspond to the canonical basis, i.e. the holomorphic point basis (normalized so that $\eta=1)$. Then the central charge has a definite value $\Delta_{a b}=2[W(a)-W(b)]$, and (A.5) follows from the definition of $C_{\tau}$ and the obvious identity

$$
\left(\bar{C}_{\bar{i}}\right)_{a b}=\operatorname{Tr}_{a b}(-1)^{F} \bar{\phi}_{\bar{i}} e^{-\beta H} .
$$

However, the canonical basis is not the natural one from a 'thermodynamical' viewpoint. In this framework one decomposes the Hilbert space $\mathcal{H}$ into sectors for which $\Delta$ and $\bar{\Delta}$ have a definite value. Such sectors should exist on general grounds. Now, whereas it is manifest that the canonical boundary conditions give a definite value for $\Delta$, it seems unlikely that they also have a definite $\bar{\Delta}$. Roughly speaking, the natural boundary conditions should correspond to the 'real' point basis for vacua, defined by prescribing the asymptotical value of the scalar fields to to be a classical vacuum 15 . We have two comments: First the identity above, being covariant under changes of bases, should be valid even in such a 'real' basis. Second, in the simplest situations we can explicitly construct the 'real' point basis and check the consistency of our formal manipulations. At any rate it would be worthwhile understanding the real point basis more clearly.

\footnotetext{
14 At least for a generic superpotential, these equations fix the curvature unambiguously.

15 This can be made more precise by defining the 'real' point vacua by starting from a large circle to quantize the theory where the point basis is unambiguous and adiabatically change the radius of the circle.
} 
The new proof of $\mathrm{tt}^{*}$ consists in showing that Eq.(A.2) and (A.3) follows from the representation (A.1) of $Q$ and the 'AB argument'. At the formal level we have (using the 'AB argument')

$$
\begin{aligned}
\delta_{i} & \operatorname{Tr}_{*}(-1)^{F} F e^{-\beta H}= \\
& =i \beta L \operatorname{Tr}_{*}(-1)^{F} F\left\{Q^{-},\left[\bar{Q}-\phi_{i}\right]\right\} e^{-\beta H} \\
& =-i \beta L \operatorname{Tr}_{*}(-1)^{F}\left\{Q^{-}, \bar{Q}^{-}\right\} \phi_{i} e^{-\beta H} \\
& =-i \beta L \operatorname{Tr}_{*}(-1)^{F} \bar{\Delta} \phi_{i} e^{-\beta H},
\end{aligned}
$$

where $*$ means some sector $(a, b)$ of the Hilbert space. Clearly, in view of (A.1) and (A.5), this is the same as (A.2) provided we interpret $\delta_{i}$ as $D_{i}$, i.e. as the metric covariant derivative. This is the correct interpretation. In general we get some contribution to the derivative of $\operatorname{Tr}_{*}(-1)^{F} F \exp [-\beta H]$ from the variation of the boundary condition $*$. Such terms have a structure which allows to absorb them in the definition of the connection in $D_{i}$. This is natural, because in a sense the path integral variation should 'dress' the vacua at infinity as well to make them be the new vacua. In this interpretation, for example, what the 'AB argument' for invariance of the Witten's index discussed in section 2 really shows is that ground state metric $g$ is covariantly constant. Similarly here this suggests that we have some covariant derivative such that

$$
D_{i} \operatorname{Tr}_{*}(-1)^{F} F e^{-\beta H}=-i \beta L \operatorname{Tr}_{*}(-1)^{F} \bar{\Delta} \phi_{i} e^{-\beta H} .
$$

(And analogously for $\bar{D}_{\bar{i}}$ ). The connection cannot be trivial. Indeed, as shown above, we can use the resulting equation to compute its curvature which turns out to be nonvanishing. It remains to show that the connection predicted by this argument is the metric one. Indeed the AB argument predicts

$$
D_{i} \operatorname{Tr}_{*}(-1)^{F} e^{-\beta H}=\bar{D}-\operatorname{Tr}_{*}(-1)^{F} e^{-\beta H}=0,
$$

for the connection induced by the variation of the boundary condition $*$.

The same reasoning applied to (A.6) gives

$$
\begin{gathered}
D_{i}\left(\bar{C}_{\bar{j}}\right)_{*}=D_{i} \operatorname{Tr}_{*}(-1)^{F} \bar{\phi}_{\bar{j}} e^{-\beta H}=-i \beta L \operatorname{Tr}_{*}(-1)^{F} \bar{\phi}_{\bar{j}}\left\{Q^{-},\left[\bar{Q}^{-}, \phi_{i}\right]\right\} e^{-\beta H}=0 \\
D_{i}\left(C_{j}\right)_{*}=D_{i} \operatorname{Tr}_{*}(-1)^{F} \phi_{j} e^{-\beta H}=-i \operatorname{Tr}_{*}(-1)^{F} \phi_{j}\left\{Q^{-},\left[\bar{Q}^{-}, \phi_{i}\right]\right\} e^{-\beta H}=D_{j}\left(C_{i}\right)_{*},
\end{gathered}
$$

showing (A.4). 
Finally we show (A.3). By definition, one has (as $L \rightarrow+\infty)$

$$
\begin{aligned}
{\left[Q, C_{j}\right]_{*} } & =\frac{i \beta}{L} \operatorname{Tr}_{*}(-1)^{F} F e^{-\beta H}\left[\phi_{j}(x=+L / 2)-\phi_{j}(x=-L / 2)\right] \\
& =\frac{i \beta}{L} \operatorname{Tr}_{*}(-1)^{F} F e^{-\beta H} \int_{-L / 2}^{+L / 2} d_{x} \phi_{j} .
\end{aligned}
$$

For $L$ large, we can replace

$$
\int_{-L / 2}^{+L / 2} d_{x} \phi_{j} \rightarrow \frac{i L}{2}\left\{Q^{+},\left[Q^{-}, \phi_{j}\right]\right\}-\frac{i L}{2}\left\{\bar{Q}^{+},\left[\bar{Q}^{-}, \phi_{j}\right]\right\}
$$

In this way we get an expression to which we can apply the 'AB argument'. One gets

$$
\begin{aligned}
{\left[Q, C_{j}\right]_{*} } & =\frac{i \beta}{2} \operatorname{Tr}_{*}(-1)^{F} e^{-\beta H}\left[\left\{Q^{+}, Q^{-}\right\}+\left\{\bar{Q}^{+}, \bar{Q}^{-}\right\}\right] \phi_{j} \\
& =-\beta \operatorname{Tr}_{*}(-1)^{F} e^{-\beta H} H \phi_{j}=\beta D_{\beta}\left[\operatorname{Tr}_{*}(-1)^{F} \phi_{j} e^{-\beta H}\right] .
\end{aligned}
$$

In view of (A.4) and dimensional analysis (i.e. dependence of the fields on the scale) this equation is equivalent to (A.3). Indeed, this is the covariant version of the statement (true only in special 'gauges') that $Q$ is the connection in the $\tau$ direction. To compare with computations done in such special gauges recall that $\beta=e^{\tau / 2+\tau^{*} / 2}$ and so $\beta g \partial_{\beta} g^{-1}=$ $2 g \partial_{\tau} g^{-1}$. 


\section{References}

[1] E. Witten, Nucl. Phys. B202 (1982) 253

[2] W. Lerche, C. Vafa, N. Warner, Nucl. Phys. B324 (1989) 427

[3] S. Cecotti and C. Vafa, Nucl. Phys. B367 (1991) 359

[4] E. Witten, Nucl. Phys. B340 (1990) 281

[5] T.Eguchi and S.K. Yang, Mod. Phys. Lett. A5 (1990) 1693

[6] C. Vafa, Mod. Phys. Lett. A6 (1991) 337

[7] S. Cecotti, L. Girardello and A. Pasquinucci, Int. J. Mod. Phys. A6 (1991) 2427

[8] S. Cecotti, Int. J. Mod. Phys. A6 (1991) 1749;

S. Cecotti, Nucl. Phys. B355 (1991) 755

[9] A.B. Zamolodchikov and Al.B. Zamolodchikov, Ann. Phys. 120 (1980) 253

[10] Al.B. Zamolodchikov, Nucl. Phys. B342 (1990) 695

[11] P. Fendley and K. Intriligator, Nucl. Phys. B372 (1992) 533.

[12] P. Fendley and K. Intriligator, Scattering and thermodynamics in integrable $N=2$ theories, BUHEP-92-5,HUTP-91/A067, to appear in Nucl. Phys. B

[13] C. Imbimbo and S. Mukhi, Nucl. Phys. B247 (1984) 471

[14] C. Callias, Commun. Math. Phys. 62 (1978)213;

R. Bott and R. Seeley, Commun. Math. Phys. 62 (1978) 235

[15] E. Witten and D. Olive, Phys. Lett. 78B (1978) 97

[16] M.Reed and B. Simon, Methods of Modern Mathematical Physics, Academic Press (NY) 1979

[17] S.J. Gates et al, Superspace: One Thousand and One Lessons in Supersymmetry (Benjamin-Cummings, Reading, 1983)

[18] L. Alvarez-Gaume and D. Freedman, Commun. Math. Phys. 80 (1981) 443

[19] R. Jackiw and C. Rebbi, Phys. Rev. D13 (1976) 3398

[20] J. Goldstone and F. Wilczek, Phys. Rev. Lett. 47 (1981) 986

[21] A.J. Niemi and G.W. Semenoff, Phys. Rep. 135 (1986) 99

[22] R. Kaul and R. Rajaraman, Phys. Lett. 131B (1983) 357

[23] R. Dijkgraaf, E. Verlinde, and H. Verlinde, Nucl. Phys. B352 (1991) 59

[24] B. Dubrovin, Geometry and integrability of topological-antitopological fusion, Napoli preprint INFN-8/92-DSF

[25] W. Leaf-Herrmann, Harvard preprint HUTP-91-A061, and to appear.

[26] E. Martinec, Phys. Lett. 217B (1989) 431;

C. Vafa and N.P. Warner, Phys. Lett. 218B (1989) 51

[27] A.B. Zamolodchikov, JETP Lett. 43 (1986) 730

[28] L. Landau and E. Lifshitz, Statistical Physics (Pergamon, New York, 1980), section 77

[29] T.R. Klassen and E. Melzer, Nucl. Phys. B350 (1990) 635 
[30] A.B. Zamolodchikov Sov. J. Nucl. Phys. 44 (1986) 529;

D. Kastor, E. Martinec and S. Shenker, Nucl. Phys. B316 (1989) 590

[31] P. Fendley, S.D. Mathur, C. Vafa and N.P. Warner, Phys. Lett. B243 (1990) 257

[32] P. Fendley, W. Lerche, S.D. Mathur and N.P. Warner, Nucl. Phys. B348 (1991) 66

[33] A. LeClair, D. Nemeschansky, and N. Warner, to appear

[34] G. Waterson, Phys. Lett. B171 (1986) 77

[35] D. Bernard and A. LeClair, Phys. Lett. 247B (1990) 309

[36] B. McCoy, J. Tracy, and T.T. Wu, J. Math. Phys. 18 (1977) 1058

[37] J. Cardy and G. Mussardo, Nucl. Phys. B 340 (1990) 387

[38] K. Kobayashi and T. Uematsu, preprint KUCP-41 (1991), hepth\#9112043

[39] P. Fendley and H. Saleur, Boston and Yale preprint BUHEP-92-15, YCTP-P13-1992

[40] P. Fendley, Boston preprint BUHEP-91-16, to appear in Nucl Phys. B

[41] Al.B. Zamolodchikov, Resonance factorized scattering and roaming trajectories, Ecole Normale preprint ENS-LPS-355

[42] P. Mathieu and M. Walton, Phys. Lett. 254B (1991) 106.

[43] D. Gepner, Fusion rings and geometry, preprint NSF-ITP-90-184

[44] A.B. Zamolodchikov, Landau Institute preprint, September 1989

[45] K.S. Narain, Nucl. Phys. B243 (1984) 131

[46] S. Cecotti and C. Vafa, Phys. Rev. Lett. 68 (1992) 903

[47] E. Abdalla, M.C. Abdalla, and M. Gomes, Phys. Rev. D25 (1982) 425; Phys. Rev. D27 (1983) 825

[48] K. Intriligator, Mod. Phys. Lett. A6 (1991) 3543

[49] D. Nemeschansky and N. Warner, USC preprint USC-91-031

[50] D. Gepner and A. Schwimmer, Weizmann preprint WIS-92/34/March-Ph, hepth\#9204020

[51] M. Bourdeau, E. J. Mlawer, H. Riggs, and H.J. Schnitzer, Brandeis preprint BRXTH-327 (1991)

[52] E. Witten, Commun. Math. Phys. 117 (1988) 353

[53] S. Cecotti and C. Vafa, work in progress.

[54] L.J. Dixon, V.S. Kaplunovsky and J. Louis, Nucl. Phys. B 355 (1991) 649.

[55] I.Antoniadis, E. Gava and K.S. Narain, preprints IC/92/50; IC/92/51.

[56] D.B. Ray and I.M. Singer, Adv. Math. 7 (1971) 145; Ann. Math. 98 (1973) 154 